EwA ZADRZYŃskA and WoJCIECH M. ZAJĄCZKOWski (Warszawa)

\title{
SOLVABILITY OF THE STATIONARY STOKES SYSTEM IN SPACES $H_{-\mu}^{2}, \mu \in(0,1)$
}

Abstract. We consider the stationary Stokes system with slip boundary conditions in a bounded domain. Assuming that data functions belong to weighted Sobolev spaces with weights equal to some power of the distance to some distinguished axis, we prove the existence of solutions to the problem in appropriate weighted Sobolev spaces.

1. Introduction. We consider the following problem for the Stokes system:

$$
\begin{array}{ll}
-\Delta v+\nabla p=f & \text { in } \Omega, \\
\operatorname{div} v=g & \text { in } \Omega, \\
v \cdot \bar{n}=0, \bar{n} \mathbb{D}(v) \bar{\tau}_{\alpha}=0, \quad \alpha=1,2, & \text { on } \partial \Omega,
\end{array}
$$

where $v=v(x)=\left(v_{1}(x), v_{2}(x), v_{3}(x)\right)$ is the velocity and $p=p(x)$ is the pressure, $\Omega=\Omega_{*} \cap D, D=\left\{x=\left(x_{1}, x_{2}, x_{3}\right): 0<r<\infty, 0<\phi<2 \pi\right.$, $\left.x_{3} \in \mathbb{R}\right\} ; r, \phi, x_{3}$ are cylindrical coordinates, i.e. $x_{1}=r \cos \phi, x_{2}=r \sin \phi$; $\Omega_{*} \subset \mathbb{R}^{3}$ is a bounded domain with boundary $\partial \Omega_{*}, \partial \Omega=\partial \Omega_{*} \backslash \gamma_{0}, \gamma_{0}=$ $\partial \Omega_{*} \cap\left\{\left(x_{1}, x_{2}, x_{3}\right): 0<r<\infty, \phi=0, x_{3} \in \mathbb{R}\right\}$. Moreover, $\bar{n}$ is the unit outward vector normal to $\partial \Omega$ and $\bar{\tau}_{\alpha}, \alpha=1,2$, is a unit tangent vector to $\partial \Omega$.

We add to the system (1.1) the following conditions:

$$
\begin{aligned}
& \left.v\right|_{\Gamma_{0}}=\left.v\right|_{\Gamma_{2 \pi}}, \\
& \left.\bar{n} \mathbb{T}(v, p)\right|_{\Gamma_{0}}=-\left.\bar{n} \mathbb{T}(v, p)\right|_{\Gamma_{2 \pi}},
\end{aligned}
$$

where $\Gamma_{0}=\Omega_{*} \cap\left\{\left(x_{1}, x_{2}, x_{3}\right): 0<r<\infty, \phi=0, x_{3} \in \mathbb{R}\right\}, \Gamma_{2 \pi}=\Omega_{*} \cap$ $\left\{\left(x_{1}, x_{2}, x_{3}\right): 0<r<\infty, \phi=2 \pi, x_{3} \in \mathbb{R}\right\}, \mathbb{T}(v, p)=\mathbb{D}(v)-p I, \mathbb{D}(v)=$ $\left\{v_{i, x_{j}}+v_{j, x_{i}}\right\}_{i, j=1,2,3}, I$ is the unit matrix.

2010 Mathematics Subject Classification: 35Q35, 35J99, 76D07.

Key words and phrases: stationary Stokes system, weighted Sobolev spaces, existence in weighted Sobolev spaces, slip boundary conditions. 
We assume that

$$
\int_{\Omega} g d x=0 .
$$

We also assume the following conditions on $\Omega_{*}$ :

(1.4) $\Omega_{*}$ contains a distinguished axis $L$ which is perpendicular to $\partial \Omega_{*}$; $L=\{x: r=0\}$

(1.5) there exist neighbourhoods of points $x^{(1)}, x^{(2)} \in L \cap \partial \Omega_{*}$ in $\partial \Omega_{*}$ which are flat, i.e. they are contained in the tangent planes at $x^{(i)}$, $i=1,2$, to $\partial \Omega_{*}$.

The aim of the paper is to prove the solvability of problem (1.1)-(1.2) in weighted Sobolev spaces $H_{-\mu}^{2}(\Omega), \mu \in(0,1)$. To formulate the main result we introduce a global Cartesian system $\left(x_{1}, x_{2}, x_{3}\right)$ in $\mathbb{R}^{3}$ such that $L$ is the $x_{3}$ axis.

Let $\Omega \subset \mathbb{R}^{3}$ be a domain. Then we define the spaces:

$$
\begin{aligned}
L_{2,-\mu}(\Omega) & =\left\{u:\|u\|_{L_{2,-\mu}(\Omega)}=\left(\int_{\Omega} u^{2}\left|x^{\prime}\right|^{-2 \mu} d x\right)^{1 / 2}<\infty\right\}, \\
H_{-\mu}^{1}(\Omega) & =\left\{u:\|u\|_{H_{-\mu}^{1}(\Omega)}=\left[\int_{\Omega}\left(u_{, x}^{2}\left|x^{\prime}\right|^{-2 \mu}+u^{2}\left|x^{\prime}\right|^{-2(\mu+1)}\right) d x\right]^{1 / 2}<\infty\right\}, \\
H_{-\mu}^{2}(\Omega) & =\left\{u:\|u\|_{H_{-\mu}^{2}(\Omega)}=\left[\int _ { \Omega } \left(u_{, x x}^{2}\left|x^{\prime}\right|^{-2 \mu}+u_{, x}^{2}\left|x^{\prime}\right|^{-2(\mu+1)}\right.\right.\right. \\
& \left.\left.\left.+u^{2}\left|x^{\prime}\right|^{-2(\mu+2)}\right) d x\right]^{1 / 2}<\infty\right\}, \quad \mu \in \mathbb{R} .
\end{aligned}
$$

In the above definitions $x=\left(x_{1}, x_{2}, x_{3}\right) \in \mathbb{R}^{3}, x^{\prime}=\left(x_{1}, x_{2}\right)$. Moreover, we do not distinguish vector and scalar-valued functions. Let $u=\left(u_{1}, \ldots, u_{n}\right)$. Then $u^{2}=\sum_{i=1}^{n} u_{i}^{2}, u_{, x}^{2}=\sum_{i=1}^{n} \sum_{j=1}^{3} u_{i, x_{j}}^{2}, u_{, x x}^{2}=\sum_{i=1}^{n} \sum_{j, k=1}^{3} u_{i, x_{j} x_{k}}^{2}$.

Let $V$ be the closure of the set $\left\{g \in H^{1}(\Omega): g\right.$ vanishes in a neighbourhood of $L$ and $\left.\int_{\Omega} g d x=0\right\}$ in $H_{-\mu}^{1}(\Omega)$.

Now, we can formulate the main result of the paper.

Main Theorem. Let $\mu \in(0,1), \partial \Omega_{*} \in C^{2}, f \in L_{2,-\mu}(\Omega), g \in V$. Moreover, let conditions (1.3)-(1.5) be satisfied. Then there exists a unique solution $v \in H_{-\mu}^{2}(\Omega), p \in H_{-\mu}^{1}(\Omega)$ of problem (1.1)-(1.2) and

$$
\|v\|_{H_{-\mu}^{2}(\Omega)}+\|p\|_{H_{-\mu}^{1}(\Omega)} \leq c\left(\|f\|_{L_{2,-\mu}(\Omega)}+\|g\|_{H_{-\mu}^{1}(\Omega)}\right) .
$$

We prove the Main Theorem in several steps. First, in Section 3 we consider the problem 


$$
\begin{array}{ll}
-\Delta v+\nabla p=f & \text { in } \mathbb{R}^{3} \backslash \bar{C}_{\delta_{0}}, \\
\operatorname{div} v=g & \text { in } \mathbb{R}^{3} \backslash \bar{C}_{\delta_{0}}, \\
v=0 & \text { on } \partial C_{\delta_{0}}, \\
v \rightarrow 0 & \text { as }|x| \rightarrow \infty,
\end{array}
$$

where $C_{\delta_{0}}=\left\{x \in \mathbb{R}^{3}: 0<\left|x^{\prime}\right|<\delta_{0}\right\}, \delta_{0}<R / 2, R>0, f \in L_{2,-\mu}\left(\mathbb{R}^{3} \backslash \bar{C}_{\delta_{0}}\right)$, $g \in H_{-\mu}^{1}\left(\mathbb{R}^{3} \backslash \bar{C}_{\delta_{0}}\right)$, supp $g \subset\left(\mathbb{R}^{3} \backslash \bar{C}_{\delta_{0}}\right) \cap C_{R, a}\left(x_{3}^{0}\right), C_{R, a}\left(x_{3}^{0}\right)=\left\{x \in \mathbb{R}^{3}:\right.$ $\left.\left|x^{\prime}\right|<R,\left|x_{3}-x_{3}^{0}\right|<a\right\}, x^{0}=\left(0,0, x_{3}^{0}\right) \in L$ is a point. We show that under the assumption that $(v, p) \in H_{-\mu}^{2}\left(\mathbb{R}^{3} \backslash \bar{C}_{\delta_{0}}\right) \times H_{-\mu}^{1}\left(\mathbb{R}^{3} \backslash \bar{C}_{\delta_{0}}\right)$ with $\operatorname{supp} v \subset\left(\mathbb{R}^{3} \backslash \bar{C}_{\delta_{0}}\right) \cap C_{R, a}\left(x_{3}^{0}\right), \operatorname{supp} p \subset\left(\mathbb{R}^{3} \backslash \bar{C}_{\delta_{0}}\right) \cap C_{R, a}\left(x_{3}^{0}\right)$ is a solution to system (1.7), the following estimate holds:

$$
\begin{aligned}
\|v\|_{H_{-\mu}^{2}\left(\mathbb{R}^{3} \backslash \bar{C}_{\delta_{0}}\right)}+\|p\|_{H_{-\mu}^{1}\left(\mathbb{R}^{3} \backslash \bar{C}_{\delta_{0}}\right)} & \leq c\left(\|f\|_{L_{2,-\mu}\left(\mathbb{R}^{3} \backslash \bar{C}_{\delta_{0}}\right)}+\|g\|_{H_{-\mu}^{1}\left(\mathbb{R}^{3} \backslash \bar{C}_{\delta_{0}}\right)}\right), \\
& \leq
\end{aligned}
$$

where $c>0$ does not depend on $\delta_{0}$.

Estimate (1.8) is derived by applying the Fourier transform with respect to the variable $x_{3}$ and by using the properties of two-dimensional equations with a parameter (see Section 2).

In Section 4 we first consider problem (1.1) in $\Omega \backslash \bar{C}_{\delta}$ with slip boundary conditions on $\partial\left(\Omega \backslash \bar{C}_{\delta}\right)$ and with data $f_{\delta} \in L_{2,-\mu}\left(\Omega \backslash \bar{C}_{\delta}\right)$ and $g_{\delta} \in H_{-\mu}^{1}(\Omega \backslash$ $\bar{C}_{\delta}$ ) such that their extensions by zero to $\Omega, \bar{f}_{\delta} \in L_{2,-\mu}(\Omega)$ and $\bar{g}_{\delta} \in H_{-\mu}^{1}(\Omega)$, approximate $f$ and $g$ in $L_{2,-\mu}(\Omega)$ and $H_{-\mu}^{1}(\Omega)$, respectively. For a solution $\left(v_{\delta}, p_{\delta}\right) \in H^{2}\left(\Omega \backslash \bar{C}_{\delta}\right) \times H^{1}\left(\Omega \backslash \bar{C}_{\delta}\right)$ of such a problem we prove the estimate

$$
\left\|v_{\delta}\right\|_{H_{-\mu}^{2}\left(\Omega \backslash \bar{C}_{\delta}\right)}+\left\|p_{\delta}\right\|_{H_{-\mu}^{1}\left(\Omega \backslash \bar{C}_{\delta}\right)} \leq c\left(\left\|f_{\delta}\right\|_{L_{2,-\mu}\left(\Omega \backslash \bar{C}_{\delta}\right)}+\left\|g_{\delta}\right\|_{H_{-\mu}^{1}\left(\Omega \backslash \bar{C}_{\delta}\right)}\right)
$$

with a constant $c>0$ independent of $\delta$.

To derive estimate (1.9) we localize the problem in neighbourhoods of four types of points:

(a) near an interior point of $L$;

(b) near a point where $L$ meets $\partial \Omega_{*}$;

(c) near an interior point of $\Omega$ but at a positive distance from $L$;

(d) near a point of $\partial \Omega$ at a positive distance from $L$.

In cases (a) and (b) solutions of localized problems satisfy estimate (1.8). In cases (c) and (d) we obtain similar estimates to (1.8) by using the results in non-weighted Sobolev spaces (see [6]) and the equivalence of $H^{k}$ and $H_{-\mu}^{k}$ norms $(k=1,2)$.

As a consequence of the above four cases and using a partition of unity we get (1.9). 
Next, we show that $\left(\bar{v}_{\delta}, \bar{p}_{\delta}\right) \in H_{\mu}^{2}(\Omega) \times H_{-\mu}^{1}(\Omega)$ where $\bar{v}_{\delta}$ and $\bar{p}_{\delta}$ are the extensions by zero of $v_{\delta}$ and $p_{\delta}$ to $\Omega$ and $\left(\bar{v}_{\delta}, \bar{p}_{\delta}\right)$ satisfies

$$
\left\|\bar{v}_{\delta}\right\|_{H_{-\mu}^{2}(\Omega)}+\left\|\bar{p}_{\delta}\right\|_{H_{-\mu}^{1}(\Omega)} \leq c\left(\left\|\bar{f}_{\delta}\right\|_{L_{2,-\mu}(\Omega)}+\left\|\bar{g}_{\delta}\right\|_{H_{-\mu}^{1}(\Omega)}\right) .
$$

The above inequality implies the convergence of $\left(\bar{v}_{\delta}, \bar{p}_{\delta}\right)$ to a function $(v, p) \in$ $H_{-\mu}^{2}(\Omega) \times H_{-\mu}^{1}(\Omega)$ which is a solution to problem (1.1)-(1.2) and satisfies estimate (1.6).

A slight modification of the proof suffices to obtain the Main Theorem in the case of $\Omega$ being a cylinder with a lateral surface of class $C^{2}$.

Now, we will review previous results concerning the solvability of the stationary Stokes system in different function spaces.

The stationary three-dimensional Stokes system has been treated before in the usual Sobolev and Hölder spaces in the papers [4, 6, 8, 9, 10, and in the book [2]. More precisely, in [4, 6] it is proved that there exists a solution $v \in W_{r}^{l+2}(\Omega), \nabla p \in W_{r}^{l}(\Omega), r>1, l \geq 0$, of system (1.1) with $g=0$ in a bounded domain $\Omega \subset \mathbb{R}^{3}$ and with the boundary condition $\left.u\right|_{\partial \Omega}=a$ under the assumptions that $f \in W_{r}^{l}(\Omega)$ and $a \in W_{r}^{l+2-1 / r}(\partial \Omega)$.

Analogous solvability results in Hölder spaces $C^{l+\alpha+2}(\bar{\Omega}), l \geq 0,0<\alpha<1$, can be found in [4, 6, 10]. In [5] the solvability of the nonstationary Stokes system with nonhomogeneous Dirichlet boundary conditions was proved in the case of nonzero $g$. In [7] the nonstationary system (1.1) with the Neumann boundary condition $\left.\mathbb{T}(v, p) \bar{n}\right|_{\partial \Omega}=0$, where $\mathbb{T}(v, p)=\left\{T_{i j}(v, p)\right\}_{i, j=1,2,3}=$ $\left\{-\delta_{i j} p+\nu\left(v_{i, x_{j}}+v_{j, x_{i}}\right)\right\}_{i, j=1,2,3}$, is considered. The existence of solutions $(v, p)$ either with $v \in W_{2}^{2}(\Omega), \nabla p \in L_{2}(\Omega)$ under the assumption that $f \in L_{2}(\Omega)$, or $v \in C^{2+\alpha}(\Omega), \nabla p \in C^{\alpha}(\Omega)$ under the assumption that $f \in L_{2}(\Omega) \cap$ $C^{\alpha}(\Omega)$, is obtained.

Solvability results in the space $W_{2}^{2}(\Omega)$ in the case of some other boundary conditions and in the case of an unbounded domain $\Omega$ were given by Solonnikov and Schadilov [9].

In contrast to the proof of the Main Theorem, the proofs of the above mentioned existence results base on the technique of potentials.

In 8] Solonnikov examines the solvability of (1.1) in weighted Sobolev spaces $H_{\mu}^{2}, \mu \in(0,1)$, in a domain $D_{\theta}=d_{\theta} \times \mathbb{R}$, where $d_{\theta}=\left\{x \in \mathbb{R}^{2}: 0<\right.$ $\phi<\theta\}$ is an angle such that $\theta \in(0,2 \pi)$. Boundary conditions imposed on the boundary of $D_{\theta}$ correspond to the boundary conditions assumed for a free boundary problem for the stationary Navier-Stokes equations. Moreover, the weight in [8] is the distance from the edge of $D_{\theta}$.

The only paper concerning the solvability of both the stationary and nonstationary Stokes system in the weighted Sobolev spaces with $\mu \in \mathbb{R} \backslash \mathbb{Z}$ is [11], where the two-dimensional Stokes system is considered; we use the results of [11] in Sections 3 and 4. 
Moreover, the results of [11] are used in [13], where the estimate and the existence for solutions of the initial-boundary value problem to the nonstationary Stokes system with slip boundary conditions are proved.

The present paper is divided into four sections. In Section 2 some auxiliary lemmas are formulated. Section 3 is devoted to deriving estimate (1.3), and Section 4 contains the proof of the Main Theorem.

2. Auxiliary lemmas. In Section 3 (see Corollary 3.5) we derive some inequality for a solution $(v, p) \in H_{-\mu}^{2}\left(\mathbb{R}^{3}\right) \times H_{-\mu}^{1}\left(\mathbb{R}^{3}\right)$ of the problem

$$
\begin{array}{ll}
-\Delta v+\nabla p=f & \text { in } \mathbb{R}^{3}, \\
\operatorname{div} v=g & \text { in } \mathbb{R}^{3}, \\
v \rightarrow 0 & \text { as }|x| \rightarrow \infty, \\
\left.v\right|_{\Gamma_{0}}=\left.v\right|_{\Gamma_{2 \pi}}, & \\
\left.\bar{n} \mathbb{T}(v, p)\right|_{\Gamma_{0}}=-\left.\bar{n} \overline{\mathbb{T}}(v, p)\right|_{\Gamma_{2 \pi}} .
\end{array}
$$

Let us denote

$$
\tilde{u}\left(x^{\prime}, \xi\right) \equiv F u\left(x^{\prime}, \xi\right)=\int_{\mathbb{R}} e^{-i x_{3} \xi} u\left(x^{\prime}, x_{3}\right) d x_{3} .
$$

Then applying the transform $F$ to problem (2.1) we get

$$
\begin{array}{ll}
-\Delta^{\prime} \tilde{v}^{\prime}+\nabla^{\prime} \tilde{p}=\tilde{f}^{\prime}-\xi^{2} \tilde{v}^{\prime} \equiv \tilde{h}^{\prime} & \text { in } \mathbb{R}^{2} \text { for a.e. } \xi \in \mathbb{R}, \\
\operatorname{div}^{\prime} \tilde{v}^{\prime}=-i \xi \tilde{v}_{3}+\tilde{g} \equiv \tilde{k} & \text { in } \mathbb{R}^{2} \text { for a.e. } \xi \in \mathbb{R}, \\
\tilde{v}^{\prime} \rightarrow 0 & \text { as }\left|x^{\prime}\right| \rightarrow \infty
\end{array}
$$

and

$$
\begin{array}{ll}
-\Delta^{\prime} \tilde{v}_{3}=\tilde{f}_{3}-\xi^{2} \tilde{v}_{3}+i \xi \tilde{\phi} \equiv \tilde{h}_{3} & \text { in } \mathbb{R}^{2} \text { for a.e. } \xi \in \mathbb{R}, \\
\tilde{v}_{3} \rightarrow 0 & \text { as }\left|x^{\prime}\right| \rightarrow \infty,
\end{array}
$$

where $\Delta^{\prime}=\partial_{x_{1}}^{2}+\partial_{x_{2}}^{2}, \nabla^{\prime}=\left(\partial_{x_{1}}, \partial_{x_{2}}\right), \tilde{u}^{\prime}=\left(\tilde{u}_{1}, \tilde{u}_{2}\right), \tilde{u} \in\{\tilde{v}, \tilde{f}, \tilde{h}\}, \operatorname{div}^{\prime} \tilde{v}=$ $\tilde{v}_{1, x_{1}}+\tilde{v}_{2, x_{2}}$. We treat $(2.2)$ and $(2.3)$ as two-dimensional problems with a parameter $\xi$.

Lemma 2.1 (see [11, Lemma 2.4]). Let $\mu \in \mathbb{R} \backslash \mathbb{Z}, \delta_{0}>0, \tilde{h}^{\prime} \in L_{2,-\mu}\left(\mathbb{R}^{3}\right)$, $\tilde{k} \in H_{-\mu}^{1}\left(\mathbb{R}^{3}\right)$. Let $\tilde{v}^{\prime}(\cdot, \xi) \in H_{-\mu}^{2}\left(\mathbb{R}^{2}\right), \tilde{p}(\cdot, \xi) \in H_{-\mu}^{1}\left(\mathbb{R}^{2}\right)$ be for almost all $\xi \in$ $\mathbb{R}$ a solution of problem (2.1). Moreover, assume that $\tilde{v}^{\prime} \in L_{2}\left(\mathbb{R} ; H_{-\mu}^{2}\left(\mathbb{R}^{2}\right)\right)$, $\tilde{p} \in L_{2}\left(\mathbb{R} ; H_{-\mu}^{1}\left(\mathbb{R}^{2}\right)\right)$. Then

$$
\left\|\tilde{v}^{\prime}\right\|_{L_{2}\left(\mathbb{R} ; H_{-\mu}^{2}\left(\mathbb{R}^{2}\right)\right)}+\|\tilde{p}\|_{L_{2}\left(\mathbb{R} ; H_{-\mu}^{1}\left(\mathbb{R}^{2}\right)\right)} \leq c\left(\left\|\tilde{h}^{\prime}\right\|_{L_{2,-\mu}\left(\mathbb{R}^{3}\right)}+\|\tilde{k}\|_{H_{-\mu}^{1}\left(\mathbb{R}^{3}\right)}\right) .
$$

Similarly, we have

Lemma 2.2. Let $\mu \in \mathbb{R} \backslash \mathbb{Z}, \delta_{0}>0, \tilde{h}_{3} \in L_{2,-\mu}\left(\mathbb{R}^{3}\right)$. Let $\tilde{v}_{3}(\xi, \cdot) \in$ $H_{-\mu}^{2}\left(\mathbb{R}^{2}\right)$ be for almost all $\xi \in \mathbb{R}$ a solution of problem (2.3). Moreover, let 
$\tilde{v}_{3} \in L_{2}\left(\mathbb{R} ; H_{-\mu}^{2}\left(\mathbb{R}^{2}\right)\right)$. Then

$$
\left\|\tilde{v}_{3}\right\|_{L_{2}\left(\mathbb{R} ; H_{-\mu}^{2}\right.}\left(\mathbb{R}^{2}\right) \leq c\left\|\tilde{h}_{3}\right\|_{L_{2,-\mu}\left(\mathbb{R}^{3}\right)} .
$$

In the proof of Lemma 3.4 we consider the following auxiliary equation:

$$
\begin{array}{ll}
\Delta \phi=\operatorname{div} h & \text { in } \mathbb{R}^{3}, \\
\phi \rightarrow 0 & \text { as }|x| \rightarrow \infty .
\end{array}
$$

Lemma 2.3. Let $h \in L_{2, \mu}\left(\mathbb{R}^{3}\right), \mu \in(0,1)$. Then there exists a solution to (2.5) such that $\nabla \phi \in L_{2, \mu}\left(\mathbb{R}^{3}\right)$ and

$$
\|\nabla \phi\|_{L_{2, \mu}\left(\mathbb{R}^{3}\right)} \leq c\|h\|_{L_{2, \mu}\left(\mathbb{R}^{3}\right)} .
$$

Proof. As $h \in L_{2, \mu}\left(\mathbb{R}^{3}\right)$, there exists a sequence $\bar{h}_{\delta} \in C_{0}^{\infty}\left(\mathbb{R}^{3}\right)$ such that $\operatorname{supp} \bar{h}_{\delta} \subset \mathbb{R}^{3} \backslash \bar{C}_{\delta}$ and

$$
\bar{h}_{\delta} \rightarrow h \quad \text { in } L_{2, \mu}\left(\mathbb{R}^{3}\right) \text { as } \delta \rightarrow 0 .
$$

Consider the problem

$$
\begin{array}{ll}
\Delta \phi_{\delta}=\operatorname{div} h_{\delta} & \text { in } \mathbb{R}^{3}, \\
\phi_{\delta} \rightarrow 0 & \text { as }|x| \rightarrow \infty .
\end{array}
$$

Let $E=E(x-y)$ be the fundamental solution to equation (2.8). Then a solution of $(2.8)$ can be written in the form

$$
\phi_{\delta}(x)=\int_{\mathbb{R}^{3}} E(x-y) \operatorname{div} y h_{\delta}(y) d y=-\int_{\mathbb{R}^{3}} \nabla_{y} E(x-y) h_{\delta}(y) d y .
$$

Hence

$$
\nabla_{x} \phi_{\delta}(x)=-\int_{\mathbb{R}^{3}} \nabla_{x} \nabla_{y} E(x-y) h_{\delta}(y) d y
$$

From [1] we have the estimates

$$
\left\|\nabla \phi_{\delta}\right\|_{L_{2, \mu}\left(\mathbb{R}^{3}\right)} \leq c\left\|h_{\delta}\right\|_{L_{2, \mu}\left(\mathbb{R}^{3}\right)}, \quad\left\|\phi_{\delta}\right\|_{L_{2, \mu}\left(\mathbb{R}^{3}\right)} \leq c\left\|h_{\delta}\right\|_{L_{2, \mu}\left(\mathbb{R}^{3}\right)} .
$$

Hence

$$
\left\|\phi_{\delta}\right\|_{W_{\mu}^{1}\left(\mathbb{R}^{3}\right)} \leq c\left\|\bar{h}_{\delta}\right\|_{L_{2, \mu}\left(\mathbb{R}^{3}\right)}
$$

where

$$
\begin{aligned}
W_{\mu}^{1}(\mathbb{R})=\{u & :\|u\|_{W_{\mu}^{1}\left(\mathbb{R}^{3}\right)} \\
& \left.=\left(\int_{\mathbb{R}^{3}}\left|x^{\prime}\right|^{2 \mu}|\phi(x)|^{2} d x+\int_{\mathbb{R}^{3}}\left|x^{\prime}\right|^{2 \mu}|\nabla \phi(x)|^{2} d x\right)^{1 / 2}<\infty\right\} .
\end{aligned}
$$

The solution $\phi_{\delta}$ of (2.8) satisfies the identity

$$
\int_{\mathbb{R}^{3}} \nabla \phi_{\delta} \nabla \psi d x=-\int_{\mathbb{R}^{3}} h_{\delta} \nabla \psi d x
$$

for all $\psi$ with $\nabla \psi \in L_{2,-\mu}(\mathbb{R})$. 
By estimate (2.9) there exists $\phi \in W_{\mu}^{1}\left(\mathbb{R}^{3}\right)$ such that

$$
\bar{\phi}_{\delta} \rightarrow \phi \quad \text { in } W_{\mu}^{1}\left(\mathbb{R}^{3}\right) \text { as } \delta \rightarrow 0
$$

and $\phi$ satisfies identity (2.10). Therefore, $\phi$ is a solution of (2.5) such that $\nabla \phi \in L_{2, \mu}\left(\mathbb{R}^{3}\right)$. Moreover, $\phi$ satisfies (2.6).

Now, let $(v, p)$ be a solution of problem (1.7). Introduce the functions

$$
\bar{w}= \begin{cases}w & \text { in } \mathbb{R}^{3} \backslash \bar{C}_{\delta_{0}}, \\ 0 & \text { in } C_{\delta_{0}}\end{cases}
$$

where $w \in\{v, p, f, g\}, \bar{w} \in\{\bar{v}, \bar{p}, \bar{f}, \bar{g}\}$.

In Sections 3 and 4 we use

Lemma 2.4. Let $\mu \in(0,1)$ and let $(v, p) \in H_{-\mu}^{2}\left(\mathbb{R}^{3} \backslash \bar{C}_{\delta_{0}}\right) \times H_{-\mu}^{1}\left(\mathbb{R}^{3} \backslash \bar{C}_{\delta_{0}}\right)$ such that $\operatorname{supp} v \subset\left(\mathbb{R}^{3} \backslash \bar{C}_{\delta_{0}}\right) \cap C_{R, a}$, supp $p \subset\left(\mathbb{R}^{3} \backslash \bar{C}_{\delta_{0}}\right) \cap C_{R, a}$ be a solution of problem (1.7) with $f \in L_{2,-\mu}\left(\mathbb{R}^{3} \backslash \bar{C}_{\delta_{0}}\right), g \in H_{-\mu}^{1}\left(\mathbb{R}^{3} \backslash \bar{C}_{\delta_{0}}\right)$ such that supp $f \subset\left(\mathbb{R}^{3} \backslash \bar{C}_{\delta_{0}}\right) \cap C_{R, a}$, supp $g \subset\left(\mathbb{R}^{3} \backslash \bar{C}_{\delta_{0}}\right) \cap C_{R, a}$. Then $(\bar{v}, \bar{p}) \in H_{-\mu}^{2}\left(\mathbb{R}^{3}\right) \times H_{-\mu}^{1}\left(\mathbb{R}^{3}\right)$ and $(\bar{v}, \bar{p})$ satisfies (2.1) with $f, g$ replaced by $\bar{f}, \bar{g}$.

Proof. Set $\hat{v}_{r}=v \cdot e_{r}, \hat{v}_{\phi}=v \cdot e_{\phi}, \hat{v}_{3}=v \cdot e_{3}, \hat{p}\left(r, \phi, x_{3}\right)=p\left(x_{1}, x_{2}, x_{3}\right)$, $\hat{f}_{r}=f \cdot e_{r}, \hat{f}_{\phi}=f \cdot e_{\phi}, \hat{f}_{3}=f \cdot e_{3}, \hat{g}\left(r, \phi, x_{3}\right)=g\left(x_{1}, x_{2}, x_{3}\right), e_{r}=$ $(\cos \phi, \sin \phi, 0), e_{\phi}=(-\sin \phi, \cos \phi, 0), e_{3}=(0,0,1)$, where $r, \phi, x_{3}$ are the cylindrical coordinates.

Next, introducing the new variable $\tau=-\ln r$ we denote

$$
\begin{aligned}
& u_{r}\left(\tau, \phi, x_{3}\right)=\hat{v}_{r}\left(e^{-\tau}, \phi, x_{3}\right), \quad u_{\phi}\left(\tau, \phi, x_{3}\right)=\hat{v}_{\phi}\left(e^{-\tau}, \phi, x_{3}\right), \\
& u_{3}\left(\tau, \phi, x_{3}\right)=\hat{v}_{3}\left(e^{-\tau}, \phi, x_{3}\right), \quad q\left(\tau, \phi, x_{3}\right)=\hat{p}\left(e^{-\tau}, \phi, x_{3}\right) \text {, } \\
& h_{r}\left(\tau, \phi, x_{3}\right)=e^{-2 \tau} \hat{f}_{r}\left(e^{-\tau}, \phi, x_{3}\right), \quad h_{\phi}\left(\tau, \phi, x_{3}\right)=e^{-2 \tau} \hat{f}_{\phi}\left(e^{-\tau}, \phi, x_{3}\right) \text {, } \\
& h_{3}\left(\tau, \phi, x_{3}\right)=e^{-2 \tau} \hat{f}_{3}\left(e^{-\tau}, \phi, x_{3}\right), \quad k\left(\tau, \phi, x_{3}\right)=e^{-\tau} \hat{g}\left(e^{-\tau}, \phi, x_{3}\right) .
\end{aligned}
$$

The problem (2.1) rewritten in the variables $\tau, \phi, x_{3}$ takes the form

$$
\begin{array}{cc}
-\left(u_{r, \tau \tau}+u_{r, \phi \phi}+e^{-2 \tau} u_{r, x_{3} x_{3}}-u_{r}-2 u_{\phi, \phi}+q+q, r\right)=h_{r} \\
& \text { in }(-\infty, A) \times(0,2 \pi) \times \mathbb{R}, \\
-\left(u_{\phi, \tau \tau}+u_{\phi, \phi \phi}+e^{-2 \tau} u_{\phi, x_{3} x_{3}}-u_{\phi}+2 u_{r, \phi}-q_{, \phi}\right)=h_{\phi} & \text { in }(-\infty, A) \times(0,2 \pi) \times \mathbb{R}, \\
(2.11) \quad-\left(u_{3, \tau \tau}+u_{3, \phi \phi}+e^{-2 \tau} u_{3, x_{3} x_{3}}-e^{-\tau} q_{, x_{3}}\right)=h_{3} & \text { in }(-\infty, A) \times(0,2 \pi) \times \mathbb{R}, \\
& -u_{r, \tau}+u_{\phi, \phi}+e^{-\tau} u_{3, x_{3}}+u_{r}=k \quad \text { in }(-\infty, A) \times(0,2 \pi) \times \mathbb{R}, \\
& \left.u\right|_{\phi=0}=\left.u\right|_{\phi=2 \pi} \quad \text { in }(-\infty, A) \times \mathbb{R}, \\
\left.\left(-u_{\phi, \tau}+u_{r, \phi}-u_{\phi}\right)\right|_{\phi=0}=\left.\left(-u_{\phi, \tau}+u_{r, \phi}-u_{\phi}\right)\right|_{\phi=2 \pi} \text { in }(-\infty, A) \times \mathbb{R},
\end{array}
$$




$$
\begin{array}{lll} 
& {\left.\left[2\left(u_{\phi, \phi}+u_{r}\right)-q\right]\right|_{\phi=0}=\left.\left[2\left(u_{\phi, \phi}+u_{r}\right)-q\right]\right|_{\phi=2 \pi}} & \text { in }(-\infty, A) \times \mathbb{R}, \\
(2.11) & \left.\left(u_{\phi, x_{3}}+u_{3, \phi}\right)\right|_{\phi=0}=\left.\left(u_{\phi, x_{3}}+u_{3, \phi}\right)\right|_{\phi=2 \pi} & \text { in }(-\infty, A) \times \mathbb{R}, \\
\text { [cont.] } & \left.u\right|_{r=A}=0 & \text { in }(0,2 \pi) \times \mathbb{R}, \\
& u \rightarrow 0 & \text { as } \tau \rightarrow-\infty,
\end{array}
$$

where $A=-\ln \delta_{0}$.

Let us extend the functions $u_{r}, u_{\phi}, u_{3}, h_{r}, h_{\phi}, h_{3}, q, k$ by zero to functions $\bar{u}_{r}, \bar{u}_{\phi}, \bar{u}_{3}, \bar{h}_{r}, \bar{h}_{\phi}, \bar{h}_{3}, \bar{q}, \bar{k}$ defined in $(-\infty,+\infty) \times(0,2 \pi) \times(-\infty,+\infty)$, i.e.

$$
\bar{w}= \begin{cases}w & \text { in }(-\infty, A) \times(0,2 \pi) \times \mathbb{R} \\ 0 & \text { in }(A,+\infty) \times(0,2 \pi) \times \mathbb{R},\end{cases}
$$

where $w \in\left\{u_{r}, u_{\phi}, u_{3}, h_{r}, h_{\phi}, h_{3}, q, k\right\}, \bar{w} \in\left\{\bar{u}_{r}, \bar{u}_{\phi}, \bar{u}_{3}, \bar{h}_{r}, \bar{h}_{\phi}, \bar{h}_{3}, \bar{q}, \bar{k}\right\}$. From the assumptions it easily follows that $\bar{u}_{r}, \bar{u}_{\phi}, \bar{u}_{3} \in H^{2}(\mathbb{R} \times(0,2 \pi) \times \mathbb{R}), \bar{q} \in$ $H^{1}(\mathbb{R} \times(0,2 \pi) \times \mathbb{R})$ and $\left(\bar{u}_{r}, \bar{u}_{q}, \bar{u}_{\phi}, \bar{q}\right)$ satisfies problem $(2.11)_{1,2,3,4,5,6,7,9}$ in $\mathbb{R} \times(0,2 \pi) \times \mathbb{R}$.

Therefore, by the equivalence of $H_{-\mu}^{k}$ and $H^{k}$ norms in this case, the assertion of the lemma follows.

3. Estimates. The aim of this section is to prove the following theorem.

Theorem 3.1. Let $\mu \in(0,1)$ and let $(v, p) \in H_{-\mu}^{2}\left(\mathbb{R}^{3} \backslash \bar{C}_{\delta_{0}}\right) \times H_{-\mu}^{1}\left(\mathbb{R}^{3} \backslash\right.$ $\left.\bar{C}_{\delta_{0}}\right)$ such that $\operatorname{supp} v \subset\left(\mathbb{R}^{3} \backslash \bar{C}_{\delta_{0}}\right) \cap C_{R, a}, \operatorname{supp} p \subset\left(\mathbb{R}^{3} \backslash \bar{C}_{\delta_{0}}\right) \cap C_{R, a}$ be a solution to problem (1.7) with $f \in L_{2,-\mu}\left(\mathbb{R}^{3} \backslash \bar{C}_{\delta_{0}}\right), g \in H_{-\mu}^{1}\left(\mathbb{R}^{3} \backslash \bar{C}_{\delta_{0}}\right)$, $\operatorname{supp} f \subset\left(\mathbb{R}^{3} \backslash \bar{C}_{\delta_{0}}\right) \cap C_{R, a}, \operatorname{supp} g \subset\left(\mathbb{R}^{3} \backslash \bar{C}_{\delta_{0}}\right) \cap C_{R, a}$. Then

$$
\begin{aligned}
\|v\|_{H_{-\mu}^{2}\left(\mathbb{R}^{3} \backslash \bar{C}_{\delta_{0}}\right)}+\|p\|_{H_{-\mu}^{1}\left(\mathbb{R}^{3} \backslash \bar{C}_{\delta_{0}}\right)} & \leq c\left(\|f\|_{L_{2,-\mu}\left(\mathbb{R}^{3} \backslash \bar{C}_{\delta_{0}}\right.}+\|g\|_{H_{-\mu}^{1}\left(\mathbb{R}^{3} \backslash \bar{C}_{\delta_{0}}\right)}\right), \\
& \leq
\end{aligned}
$$

where $c>0$ is independent of $\delta_{0}$.

To prove Theorem 3.1 we need some auxiliary estimates for solutions of problem (2.1) which will be derived in Lemmas 3.2-3.4 below.

In Lemmas 3.2-3.4 below we assume that $(v, p)$ is a solution to problem (2.1) such that supp $v \subset\left(\mathbb{R}^{3} \backslash \bar{C}_{\delta_{0}}\right) \cap C_{R, a}, \operatorname{supp} p \subset\left(\mathbb{R}^{3} \backslash \bar{C}_{\delta_{0}}\right) \cap C_{R, a}$ and $\operatorname{supp} f \subset\left(\mathbb{R}^{3} \backslash \bar{C}_{\delta_{0}}\right) \cap C_{R, a}, \operatorname{supp} g \subset\left(\mathbb{R}^{3} \backslash \bar{C}_{\delta_{0}}\right) \cap C_{R, a}$.

Lemma 3.2. Let $\mu \in(0,1), \varepsilon \in(0,1)$ and let $\tilde{v}, \tilde{p}, \tilde{f}, \tilde{g}$ denote the Fourier transforms with respect to $x_{3}$ of the functions $v, p, f, g$ (see Section 2$)$. Moreover, assume that

$$
\begin{aligned}
& \int_{\mathbb{R}} d \xi \int_{\mathbb{R}^{2}} \xi^{2}|\tilde{p}|^{2}\left|x^{\prime}\right|^{-2 \mu} d x^{\prime}+\int_{\mathbb{R}} d \xi \int_{\mathbb{R}^{2}}|\tilde{v}|^{2}\left|x^{\prime}\right|^{-2 \mu-2} d x^{\prime}<\infty, \\
& \int_{\mathbb{R}} d \xi \int_{\mathbb{R}^{2}}|\tilde{f}|^{2}\left|x^{\prime}\right|^{-2 \mu} d x^{\prime}+\int_{\mathbb{R}} d \xi \int_{\mathbb{R}^{2}} \xi^{2}|\tilde{g}|^{2}\left|x^{\prime}\right|^{-2 \mu} d x^{\prime}<\infty .
\end{aligned}
$$


Then solutions $(v, p)$ of (2.1) satisfy the inequality

$$
\begin{aligned}
\int_{\mathbb{R}} d \xi \int_{\mathbb{R}^{2}} \xi^{2}\left(\left|\nabla^{\prime} \tilde{v}\right|^{2}+\xi^{2}|\tilde{v}|^{2}\right)\left|x^{\prime}\right|^{-2 \mu} d x^{\prime} \\
\leq \varepsilon \int_{\mathbb{R}} d \xi \int_{\mathbb{R}^{2}} \xi^{2}|\tilde{p}|^{2}\left|x^{\prime}\right|^{-2 \mu} d x^{\prime}+c\left(\frac{1}{\varepsilon}\right) \int_{\mathbb{R}} d \xi \int_{\mathbb{R}^{2}} \xi^{2}|\tilde{v}|^{2}\left|x^{\prime}\right|^{-2 \mu-2} d x^{\prime} \\
\quad+c\left(\frac{1}{\varepsilon}\right) \int_{\mathbb{R}} d \xi \int_{\mathbb{R}^{2}}\left(|\tilde{f}|^{2}+\xi^{2}|\tilde{g}|^{2}\right)\left|x^{\prime}\right|^{-2 \mu} d x^{\prime}
\end{aligned}
$$

where $c=c(1 / \varepsilon)$ is an increasing function.

Proof. Differentiate problem (2.1) with respect to $x_{3}$ and apply the Fourier transform $F$ to get

$$
\begin{array}{ll}
-i \xi \Delta^{\prime} \tilde{v}^{\prime}+i \xi^{3} \tilde{v}^{\prime}+i \xi \nabla^{\prime} \tilde{p}=i \xi f^{\prime} & \text { in } D, \\
i \xi\left(\tilde{v}_{1, x_{1}}+\tilde{v}_{2, x_{2}}\right)=-\xi^{2} \tilde{v}_{3}+i \xi \tilde{g} & \text { in } D, \\
-i \xi \Delta^{\prime} \tilde{v}_{3}+i \xi^{3} \tilde{v}_{3}=\xi^{2} \tilde{p}+i \xi \tilde{f}_{3} & \text { in } D .
\end{array}
$$

Multiplying (3.3) $)_{1}$ by $-i \xi \tilde{v}^{\prime}\left|x^{\prime}\right|^{-2 \mu}$, and $(3.3)_{3}$ by $-i \xi \tilde{v}_{3}\left|x^{\prime}\right|^{-2 \mu}$, and then integrating the results with respect to $x^{\prime}$ and $\xi$ we obtain

$$
\begin{aligned}
& \int_{\mathbb{R}} d \xi \int_{\mathbb{R}^{2}} \xi^{2}\left(\left|\nabla^{\prime} \tilde{v}\right|^{2}+\xi^{2}|\tilde{v}|^{2}\right)\left|x^{\prime}\right|^{-2 \mu} d x^{\prime} \\
& =2 \mu \int_{\mathbb{R}} d \xi \int_{\mathbb{R}^{2}} \xi^{2} \nabla^{\prime} \tilde{v} \cdot \tilde{v}\left|x^{\prime}\right|^{-2 \mu-1} \nabla^{\prime}\left|x^{\prime}\right| d x^{\prime} \\
& \quad-2 \mu \int_{\mathbb{R}} d \xi \int_{\mathbb{R}^{2}} \xi^{2} \tilde{p} \tilde{v}^{\prime} \cdot\left|x^{\prime}\right|^{-2 \mu-1} \nabla^{\prime}\left|x^{\prime}\right| d x^{\prime}+\int_{\mathbb{R}} d \xi \int_{\mathbb{R}^{2}} \xi^{2} \tilde{f} \cdot \tilde{v}\left|x^{\prime}\right|^{-2 \mu} d x^{\prime} \\
& \quad-\int_{\mathbb{R}} d \xi \int_{\mathbb{R}^{2}} \xi^{2} \tilde{g} \tilde{p}\left|x^{\prime}\right|^{-2 \mu} d x^{\prime}
\end{aligned}
$$

Hence

$$
\begin{aligned}
& \int_{\mathbb{R}} d \xi \int_{\mathbb{R}^{2}} \xi^{2}\left(\left|\nabla^{\prime} \tilde{v}\right|^{2}+\xi^{2}|\tilde{v}|^{2}\right)\left|x^{\prime}\right|^{-2 u} d x^{\prime} \\
& \leq \frac{\varepsilon}{2} \int_{\mathbb{R}} d \xi \int_{\mathbb{R}^{2}} \xi^{2}\left(\left|\nabla^{\prime} \tilde{v}\right|^{2}+\xi^{2}|\tilde{v}|^{2}\right)\left|x^{\prime}\right|^{-2 \mu} d x^{\prime}+\frac{\varepsilon}{2} \int_{\mathbb{R}} d \xi \int_{\mathbb{R}^{2}} \xi^{2}|\tilde{p}|\left|x^{\prime}\right|^{-2 \mu} d x^{\prime} \\
& \quad+c\left(\frac{1}{\varepsilon}\right) \int_{\mathbb{R}} d \xi \int_{\mathbb{R}^{2}} \xi^{2}|\tilde{v}|^{2}\left|x^{\prime}\right|^{-2 \mu-2} d x^{\prime}+c\left(\frac{1}{\varepsilon}\right) \int_{\mathbb{R}} d \xi \int_{\mathbb{R}^{2}}\left(|\tilde{f}|^{2}+\xi^{2}|\tilde{g}|^{2}\right)\left|x^{\prime}\right|^{-2 \mu} d x^{\prime} .
\end{aligned}
$$

For sufficiently small $\varepsilon$ estimate (3.2) follows.

Now, we have to estimate the first two terms on the r.h.s. of (3.2). In the next lemma we estimate the first term. 
Lemma 3.3. Let $\mu \in(0,1)$ and

$$
\begin{gathered}
\int_{\mathbb{R}} d \xi \int_{\mathbb{R}^{2}} \xi^{2}|\tilde{v}|^{2}\left|x^{\prime}\right|^{-2 \mu-2} d x^{\prime}<\infty, \\
\int_{\mathbb{R}} d \xi \int_{\mathbb{R}^{2}}|\tilde{f}|^{2}\left|x^{\prime}\right|^{-2 \mu} d x^{\prime}+\int_{\mathbb{R}} d \xi \int_{\mathbb{R}^{2}} \xi^{2}|\tilde{g}|^{2}\left|x^{\prime}\right|^{-2 \mu} d x^{\prime}+\int_{\mathbb{R}}\|\tilde{g}\|_{H_{-\mu}^{1}\left(\mathbb{R}^{2}\right)}^{2} d \xi<\infty .
\end{gathered}
$$

Then solutions of (2.1) satisfy the inequality

$$
\begin{aligned}
& \int_{\mathbb{R}}\left(\|\tilde{v}\|_{H_{-\mu}^{2}\left(\mathbb{R}^{2}\right)}^{2}+\|\tilde{p}\|_{H_{-\mu}^{1}\left(\mathbb{R}^{2}\right)}^{2}\right) d \xi \\
& \quad+\int_{\mathbb{R}} d \xi \int_{\mathbb{R}^{2}} \xi^{2}\left(\left|\nabla^{\prime} \tilde{v}\right|^{2}+\xi^{2}|\tilde{v}|^{2}\right)\left|x^{\prime}\right|^{-2 \mu} d x^{\prime} \\
& \quad+\int_{\mathbb{R}} d \xi \int_{\mathbb{R}^{2}} \xi^{2}|\tilde{p}|^{2}\left|x^{\prime}\right|^{-2 \mu} d x^{\prime} \\
& \leq c \int_{\mathbb{R}} d \xi \int_{\mathbb{R}^{2}} \xi^{2}|\tilde{v}|^{2}\left|x^{\prime}\right|^{-2 \mu-2} d x^{\prime}+c\left(\|f\|_{L_{2,-\mu}\left(\mathbb{R}^{3}\right)}^{2}+\int_{\mathbb{R}}\|\tilde{g}\|_{H_{-\mu}^{1}\left(\mathbb{R}^{2}\right)}^{2} d \xi\right) .
\end{aligned}
$$

Proof. First, we will derive an estimate for $\int_{\mathbb{R}} d \xi \int_{\mathbb{R}^{2}} \xi^{2}|\tilde{p}|^{2}\left|x^{\prime}\right|^{-2 \mu} d x^{\prime}$. To do this we use the system

$$
\begin{array}{ll}
-\Delta \phi+\nabla \eta=0 & \text { in } \mathbb{R}^{3}, \\
\operatorname{div} \phi=p\left|x^{\prime}\right|^{-\mu} & \text { in } \mathbb{R}^{3}, \\
\phi \rightarrow 0 & \text { as }|x| \rightarrow \infty .
\end{array}
$$

Solutions of (3.5) satisfy the estimate

$$
\|\nabla \phi\|_{L_{2}\left(\mathbb{R}^{3}\right)}^{2} \leq c\|p\|_{L_{2,-\mu}\left(\mathbb{R}^{3}\right)}^{2} .
$$

Substituting $\phi=\psi\left|x^{\prime}\right|^{-\mu}$ in (3.6) we get

$$
\int_{\mathbb{R}^{3}}\left[\left|\nabla^{\prime}\left(\psi\left|x^{\prime}\right|^{-\mu}\right)\right|^{2}+\left|\psi_{, x_{3}}\right|^{2}\left|x^{\prime}\right|^{-2 \mu}\right] d x \leq c \int_{\mathbb{R}^{3}}|p|^{2}\left|x^{\prime}\right|^{-2 \mu} d x^{\prime} .
$$

Differentiating system (3.5) with respect to $x_{3}$ we obtain, instead of (3.7),

$$
\int_{\mathbb{R}^{3}}\left[\left|\nabla^{\prime}\left(\psi_{, x_{3}}\left|x^{\prime}\right|^{-\mu}\right)\right|^{2}+\left|\psi_{, x_{3} x_{3}}\right|^{2}\left|x^{\prime}\right|^{-2 \mu}\right] d x \leq c \int_{\mathbb{R}^{3}}\left|p_{, x_{3}}\right|^{2}\left|x^{\prime}\right|^{-2 \mu} d x^{\prime} .
$$

Next, applying the Fourier transform $F$ yields

$$
\int_{\mathbb{R}} d \xi \int_{\mathbb{R}^{2}}\left[\xi^{2}\left|\nabla^{\prime}\left(\tilde{\psi}\left|x^{\prime}\right|^{-\mu}\right)\right|^{2}+\xi^{4}|\tilde{\psi}|^{2}\left|x^{\prime}\right|^{-2 \mu}\right] d x^{\prime} \leq c \int_{\mathbb{R}} d \xi \int_{\mathbb{R}^{2}} \xi^{2}|\tilde{p}|^{2}\left|x^{\prime}\right|^{-2 \mu} d x^{\prime} .
$$

Let $\zeta \in C_{0}^{\infty}\left(\mathbb{R}_{+}\right)$be a function such that $0 \leq \zeta(t) \leq 1$ for $t \in \mathbb{R}_{+}$, $\zeta(t)=0$ for $t \leq a / 2, \zeta(t)=1$ for $t \geq a$ and $|\dot{\zeta}| \leq c / a, a>0$.

Multiplying $(2.1)_{1}$ by $\overline{\tilde{\psi}}^{\prime}\left|x^{\prime}\right|^{-2 \mu} \zeta\left(|\xi|\left|x^{\prime}\right|\right)$ and $(2.2)$ by $\overline{\tilde{\psi}}_{3}\left|x^{\prime}\right|^{-2 \mu} \zeta\left(|\xi|\left|x^{\prime}\right|\right)$ and then integrating the result with respect to $x^{\prime}$ we get 


$$
\begin{array}{r}
\int_{\mathbb{R}^{2}}\left(-\Delta^{\prime} \tilde{v}+\xi^{2} \tilde{v}\right) \cdot \overline{\tilde{\psi}}\left|x^{\prime}\right|^{-2 \mu} \zeta d x^{\prime}+\int_{\mathbb{R}^{2}}\left(p_{, x_{1}} \overline{\tilde{\psi}}_{1}+p_{, x_{2}} \overline{\tilde{\psi}}_{2}-i \xi \tilde{\tilde{\psi}} \bar{\psi}_{3}\right)\left|x^{\prime}\right|^{-2 \mu} \zeta d x^{\prime} \\
=\int_{\mathbb{R}^{2}} \tilde{f} \overline{\tilde{\psi}}\left|x^{\prime}\right|^{-2 \mu} \zeta d x^{\prime}
\end{array}
$$

Hence

$$
\begin{aligned}
& \int_{\mathbb{R}^{2}}\left[\tilde{p}\left(\overline{\tilde{\psi}}_{1}\left|x^{\prime}\right|^{-\mu}\right)_{x_{1}}+\tilde{p}\left(\overline{\tilde{\psi}}_{2}\left|x^{\prime}\right|^{-\mu}\right)_{, x_{2}}+\tilde{p} i \overline{\xi \tilde{\psi}_{3}}\left|x^{\prime}\right|^{-\mu} \zeta\right] d x^{\prime} \\
& \quad+\int_{\mathbb{R}^{2}} \tilde{p}\left[\overline{\tilde{\psi}}_{1}\left|x^{\prime}\right|^{-\mu}\left(\left|x^{\prime}\right|^{-\mu} \zeta\right)_{, x_{1}}+\overline{\tilde{\psi}}_{2}\left|x^{\prime}\right|^{-\mu}\left(\left|x^{\prime}\right|^{-\mu} \zeta\right)_{, x_{2}}\right] d x^{\prime} \\
& =\int_{\mathbb{R}^{2}}\left[\nabla^{\prime} \tilde{v} \cdot \nabla^{\prime}\left(\overline{\tilde{\psi}}\left|x^{\prime}\right|^{-2 \mu} \zeta\right)+\xi^{2} \tilde{v} \cdot \overline{\tilde{\psi}}\left|x^{\prime}\right|^{-2 \mu}\right] d x^{\prime}-\int_{\mathbb{R}^{2}} \tilde{f} \cdot \overline{\tilde{\psi}}\left|x^{\prime}\right|^{-2 \mu} \zeta d x^{\prime}
\end{aligned}
$$

Inserting $\phi=\psi\left|x^{\prime}\right|^{-\mu}$ in $(3.5)_{2}$ and next applying the Fourier transform with respect to $x_{3}$ gives

$$
\operatorname{div}^{\prime}\left(\tilde{\psi}^{\prime}\left|x^{\prime}\right|^{-\mu}\right)-i \xi \tilde{\psi}_{3}\left|x^{\prime}\right|^{-\mu}=\tilde{p}\left|x^{\prime}\right|^{-\mu} .
$$

Therefore (3.9) implies

$$
\begin{aligned}
& \int_{\mathbb{R}} \xi^{2} d \xi \int_{\mathbb{R}^{2}}|\tilde{p}|^{2}\left|x^{\prime}\right|^{-2 \mu} \zeta d x^{\prime} \\
& \leq c \int_{\mathbb{R}} \xi^{2} d \xi \int_{\mathbb{R}^{2}}|\tilde{p}|^{2}|\tilde{\psi}|^{2}\left(\left|x^{\prime}\right|^{-2 \mu-1} \zeta+\left|x^{\prime}\right|^{-2 \mu} \dot{\zeta}|\xi|\right) d x^{\prime} \\
&+c \int_{\mathbb{R}} \xi^{2} d \xi \int_{\mathbb{R}^{2}}\left[\left|\nabla^{\prime} \tilde{v}\right|\left|\nabla^{\prime}\left(\tilde{\psi}\left|x^{\prime}\right|^{-\mu}\right)\right|\left|x^{\prime}\right|^{-\mu} \zeta\right. \\
&+\left|\nabla^{\prime} \tilde{v}\right||\tilde{\psi}|\left|x^{\prime}\right|^{-2 \mu-1} \zeta+\left|\nabla^{\prime} \tilde{v}\right||\tilde{\psi}|\left|x^{\prime}\right|^{-2 \mu}|\xi| \dot{\zeta} \\
&\left.+\xi^{2}|\tilde{v}||\tilde{\psi}|\left|x^{\prime}\right|^{-2 \mu} \zeta\right] d x^{\prime}+c \int_{\mathbb{R}} \xi^{2} d \xi \int_{\mathbb{R}^{2}}|\tilde{f}||\tilde{\psi}|\left|x^{\prime}\right|^{-2 \mu} \zeta d x^{\prime}
\end{aligned}
$$

Using the properties of the function $\zeta$ we obtain

$$
\begin{aligned}
& \int_{\mathbb{R}} \xi^{2} d \xi \int_{\mathbb{R}^{2}}|\tilde{p}|\left|x^{\prime}\right|^{-2 \mu} d x^{\prime} \leq \varepsilon_{1} \int_{\mathbb{R}} \xi^{2} d \xi \int_{\mathbb{R}^{2}}|\tilde{p}|^{2}\left|x^{\prime}\right|^{-2 \mu} \zeta d x^{\prime} \\
& \quad+c\left(\frac{1}{\varepsilon_{1}}\right) \int_{\mathbb{R}} \xi^{2} d \xi \int_{\mathbb{R}^{2}}|\tilde{\psi}|^{2}\left|x^{\prime}\right|^{-2 \mu-2} \zeta d x^{\prime}+\varepsilon_{2} \int_{\mathbb{R}} \xi^{4} d \xi \int_{\mathbb{R}^{2}}|\tilde{\psi}|^{2}\left|x^{\prime}\right|^{-2 \mu}|\dot{\zeta}| d x^{\prime} \\
& \quad+c\left(\frac{1}{\varepsilon_{2}}\right) \int_{\mathbb{R}} \xi^{2} d \xi \int_{\mathbb{R}^{2}}|\tilde{p}|^{2}\left|x^{\prime}\right|^{-2 \mu}|\dot{\zeta}| d x^{\prime} \\
& \quad+\varepsilon_{3} \int_{\mathbb{R}} \xi^{2} d \xi \int_{\mathbb{R}^{2}}\left[\left|\nabla^{\prime}\left(\tilde{\psi}\left|x^{\prime}\right|^{-\mu}\right)\right|^{2}+|\tilde{\psi}|^{2}\left|x^{\prime}\right|^{-2 \mu-2}\right] \zeta d x^{\prime}
\end{aligned}
$$




$$
\begin{aligned}
& +c\left(\frac{1}{\varepsilon_{3}}\right) \int_{\mathbb{R}} \xi^{2} d \xi \int_{\mathbb{R}^{2}}\left|\nabla^{\prime} \tilde{v}\right|^{2}\left|x^{\prime}\right|^{-2 \mu} \zeta d x^{\prime} \\
& +\varepsilon_{4} \int_{\mathbb{R}} \xi^{4} d \xi \int_{\mathbb{R}^{2}}|\tilde{\psi}|^{2}\left|x^{\prime}\right|^{-2 \mu}|\dot{\zeta}| d x^{\prime}+c\left(\frac{1}{\varepsilon_{4}}\right) \int_{\mathbb{R}} \xi^{2} d \xi \int_{\mathbb{R}^{2}}\left|\nabla^{\prime} \tilde{v}\right|^{2}\left|x^{\prime}\right|^{-2 \mu}|\dot{\zeta}| d x^{\prime} \\
& +\varepsilon_{5} \int_{\mathbb{R}} \xi^{4} d \xi \int_{\mathbb{R}^{2}}|\tilde{\psi}|^{2}\left|x^{\prime}\right|^{-2 \mu} \zeta d x^{\prime}+c\left(\frac{1}{\varepsilon_{5}}\right) \int_{\mathbb{R}} \xi^{4} d \xi \int_{\mathbb{R}^{2}}|\tilde{v}|^{2}\left|x^{\prime}\right|^{-2 \mu} \zeta d x^{\prime} \\
& +c\left(\frac{1}{\varepsilon_{5}}\right) \int_{\mathbb{R}} d \xi \int_{\mathbb{R}^{2}}|\tilde{f}|^{2}\left|x^{\prime}\right|^{-2 \mu} \zeta d x^{\prime} .
\end{aligned}
$$

Notice that $\left|x^{\prime}\right|^{-1} \leq(2 / a)|\xi|$ for $\left(x^{\prime}, \xi\right)$ such that $\left|x^{\prime}\right||\xi| \in \operatorname{supp} \zeta$. Therefore, assuming that $\varepsilon_{1}=1 / 2$ and adding $\frac{1}{2} \int_{\mathbb{R}} \xi^{2} d \xi \int_{\mathbb{R}^{2}}|\tilde{p}|^{2}\left|x^{\prime}\right|^{-2 \mu}(1-\zeta) d x^{\prime}$ to both sides of the above inequality gives

$$
\begin{aligned}
\frac{1}{2} \int_{\mathbb{R}} \xi^{2} d \xi \int_{\mathbb{R}^{2}}|\tilde{p}|^{2}\left|x^{\prime}\right|^{-2 \mu} d x^{\prime} \leq \frac{1}{2} \int_{\mathbb{R}} \xi^{2} d \xi \int_{\mathbb{R}^{2}}|\tilde{p}|^{2}\left|x^{\prime}\right|^{-2 \mu}(1-\zeta) d x^{\prime} \\
+\left(c\left(\frac{1}{\varepsilon_{1}}\right) \frac{1}{a^{2}}+\frac{c \varepsilon_{2}}{a}+\frac{c \varepsilon_{3}}{a^{2}}+\frac{c \varepsilon_{4}}{a}+c \varepsilon_{5}\right) \int_{\mathbb{R}} \xi^{4} d \xi \int_{\mathbb{R}^{2}}|\tilde{\psi}|^{2}\left|x^{\prime}\right|^{-2 \mu} d x^{\prime} \\
\quad+c \varepsilon_{3} \int_{\mathbb{R}} \xi^{2} d \xi \int_{\mathbb{R}^{2}}\left|\nabla^{\prime}\left(\tilde{\psi}\left|x^{\prime}\right|^{-\mu}\right)\right|^{2} d x^{\prime}+c\left(\frac{1}{\varepsilon_{2}}\right) \frac{1}{a} \int_{\mathbb{R}} \xi^{2} d \xi \int_{\mathbb{R}^{2}}|\tilde{p}|^{2}\left|x^{\prime}\right|^{-2 \mu} d x^{\prime} \\
+c\left(\frac{1}{\varepsilon_{3}}, \frac{1}{\varepsilon_{4}}, \frac{1}{a}\right) \int_{\mathbb{R}} \xi^{2} d \xi \int_{\mathbb{R}^{2}}\left|\nabla^{\prime} \tilde{v}\right|^{2}\left|x^{\prime}\right|^{-2 \mu} d x^{\prime} \\
+c\left(\frac{1}{\varepsilon_{5}}\right) \frac{1}{a} \int_{\mathbb{R}} \xi^{4} d \xi \int_{\mathbb{R}^{2}}|\tilde{v}|^{2}\left|x^{\prime}\right|^{-2 \mu} d x^{\prime}+c\left(\frac{1}{\varepsilon_{5}}\right) \int_{\mathbb{R}} d \xi \int_{\mathbb{R}^{2}}|\tilde{f}|^{2}\left|x^{\prime}\right|^{-2 \mu} d x^{\prime} .
\end{aligned}
$$

Since $1-\zeta \neq 0$ for $\left(\xi, x^{\prime}\right)$ such that $|\xi|<a\left|x^{\prime}\right|$, in view of (3.8) we get, for sufficiently small $\varepsilon_{i}(i=2, \ldots, 5)$ and for sufficiently large $a$,

$$
\begin{aligned}
& \int_{\mathbb{R}} \xi^{2} d \xi \int_{\mathbb{R}^{2}}|\tilde{p}|^{2}\left|x^{\prime}\right|^{-2 \mu} d x^{\prime} \leq c \int_{\mathbb{R}} d \xi \int_{\mathbb{R}^{2}}|\tilde{p}|^{2}\left|x^{\prime}\right|^{-2 \mu-2} d x^{\prime} \\
& \quad+c \int_{\mathbb{R}} \xi^{2} d \xi \int_{\mathbb{R}^{2}}\left(\left|\nabla^{\prime} \tilde{v}\right|^{2}+\xi^{2}|\tilde{v}|^{2}\right)\left|x^{\prime}\right|^{-2 \mu} d x^{\prime}+c \int_{\mathbb{R}} d \xi \int_{\mathbb{R}^{2}}|\tilde{f}|^{2}\left|x^{\prime}\right|^{-2 \mu} d x^{\prime} .
\end{aligned}
$$

Using (3.10) in (3.2) we obtain, for sufficiently small $\varepsilon$,

$$
\begin{aligned}
& \int_{\mathbb{R}} d \xi \int_{\mathbb{R}^{2}} \xi^{2}\left(\left.\nabla^{\prime} \tilde{v}\right|^{2}+\xi^{2}|\tilde{v}|^{2}\right)\left|x^{\prime}\right|^{-2 \mu} d x^{\prime} \leq c \varepsilon \int_{\mathbb{R}} d \xi \int_{\mathbb{R}^{2}}|\tilde{p}|^{2}\left|x^{\prime}\right|^{-2 \mu-2} d x^{\prime} \\
& \quad+c \int_{\mathbb{R}} d \xi \int_{\mathbb{R}^{2}} \xi^{2}|\tilde{v}|^{2}\left|x^{\prime}\right|^{-2 \mu-2} d x^{\prime}+c \int_{\mathbb{R}} d \xi \int_{\mathbb{R}^{2}}\left(|\tilde{f}|^{2}+\xi^{2}|\tilde{g}|^{2}\right)\left|x^{\prime}\right|^{-2 \mu} d x^{\prime} .
\end{aligned}
$$


Now, Lemmas 2.1-2.2 yield the estimates

$$
\begin{aligned}
\int_{\mathbb{R}}\left(\|\tilde{v}\|_{H_{-\mu}^{2}\left(\mathbb{R}^{2}\right)}^{2}+\|\tilde{p}\|_{H_{-\mu}^{1}\left(\mathbb{R}^{2}\right)}^{2}\right) d \xi \leq c \int_{\mathbb{R}}\left(\xi^{4}\left\|\tilde{v}^{\prime}\right\|_{L_{2,-\mu}\left(\mathbb{R}^{2}\right)}^{2}\right. & \\
& \left.+\xi^{2}\left\|\tilde{v}_{3}\right\|_{H_{-\mu}^{1}\left(\mathbb{R}^{2}\right)}^{2}+\|\tilde{f}\|_{L_{2,-\mu}\left(\mathbb{R}^{2}\right)}^{2}+\|\tilde{g}\|_{H_{\mu}^{1}\left(\mathbb{R}^{2}\right)}^{2}\right) d \xi
\end{aligned}
$$

and

$$
\int_{\mathbb{R}}\left\|\tilde{v}_{3}\right\|_{H_{-\mu}^{2}\left(\mathbb{R}^{2}\right)}^{2} d \xi \leq c \int_{\mathbb{R}}\left(\xi^{4}\left\|\tilde{v}_{3}\right\|_{L_{2,-\mu}\left(\mathbb{R}^{2}\right)}^{2}+\xi^{2}\|\tilde{p}\|_{L_{2,-\mu}\left(\mathbb{R}^{2}\right)}^{2}\right) d \xi .
$$

By (3.11)-(3.13) we have, for sufficiently small $\varepsilon$,

$$
\begin{aligned}
\int_{\mathbb{R}}\left(\left\|\tilde{v}^{\prime}\right\|_{H_{-\mu}^{2}\left(\mathbb{R}^{2}\right)}^{2}+\|\tilde{p}\|_{H_{-\mu}^{1}\left(\mathbb{R}^{2}\right)}^{2}\right) d \xi \leq c\left(\|f\|_{L_{2,-\mu}\left(\mathbb{R}^{3}\right)}^{2}\right. & \\
& \left.+\int_{\mathbb{R}}\|\tilde{g}\|_{H_{-\mu}^{1}\left(\mathbb{R}^{2}\right)}^{2} d \xi+\int_{\mathbb{R}} d \xi \int_{\mathbb{R}^{2}} \xi^{2}\left|\tilde{v}^{\prime}\right|^{2}\left|x^{\prime}\right|^{-2 \mu-2} d x^{\prime}\right) .
\end{aligned}
$$

Inequalities (3.10), (3.11), (3.13) and (3.14) yield (3.4). This ends the proof.

In the last step of the proof of Theorem 3.1 we need to estimate the integral $\int_{\mathbb{R}} d \xi \int_{\mathbb{R}^{2}} \xi^{2}|\tilde{v}|^{2}\left|x^{\prime}\right|^{-2 \mu-2} d x^{\prime}$. In order to do this we define

$$
\begin{aligned}
& Q_{1}=\left\{\left(x^{\prime}, \xi\right) \in \mathbb{R}^{2} \times \mathbb{R}:|\xi|\left|x^{\prime}\right| \leq a_{1}\right\}, \\
& Q_{2}=\left\{\left(x^{\prime}, \xi\right) \in \mathbb{R}^{2} \times \mathbb{R}:|\xi|\left|x^{\prime}\right| \geq a_{2}\right\}, \\
& Q_{3}=\left\{\left(x^{\prime}, \xi\right) \in \mathbb{R}^{2} \times \mathbb{R}: a_{1} \leq|\xi|\left|x^{\prime}\right| \leq a_{2}\right\},
\end{aligned}
$$

where $a_{1}, a_{2}$ are positive numbers.

Lemma 3.4. Let $\mu \in(0,1)$ and

$$
\begin{aligned}
& \int_{\mathbb{R}} d \xi \int_{\mathbb{R}^{2}}|\tilde{v}|^{2}\left|x^{\prime}\right|^{-2 \mu-4} d x^{\prime}+\int_{\mathbb{R}} d \xi \int_{\mathbb{R}^{2}} \xi^{4}|\tilde{v}|^{2}\left|x^{\prime}\right|^{-2 \mu} d x^{\prime}<\infty, \\
& \int_{\mathbb{R}} d \xi \int_{\mathbb{R}^{2}}|\tilde{f}|\left|x^{\prime}\right|^{-2 \mu} d x^{\prime}+\int_{\mathbb{R}} d \xi \int_{\mathbb{R}^{2}}\left(\left|\nabla^{\prime} \tilde{g}\right|^{2}+\xi^{2}|\tilde{g}|^{2}\right) d x^{\prime}<\infty .
\end{aligned}
$$

Moreover, assume that the assumptions of Theorem 3.1 concerning the supports of $v, p, f$ and $g$ are satisfied. Then solutions of (2.1) satisfy

$$
\begin{aligned}
\int_{\mathbb{R}} d \xi \int_{\mathbb{R}^{2}} \xi^{2}|\tilde{v}|^{2} \mid & \left.x^{\prime}\right|^{-2 \mu-2} d x^{\prime} \leq 2 a_{1}^{2} \int_{\mathbb{R}} d \xi \int_{\mathbb{R}^{2}}|\tilde{v}|^{2}\left|x^{\prime}\right|^{-2 \mu-4} d x^{\prime} \\
& +\frac{2}{a_{2}^{2}} \int_{\mathbb{R}} d \xi \int_{\mathbb{R}^{2}} \xi^{4}|\tilde{v}|^{2}\left|x^{\prime}\right|^{-2 \mu} d x^{\prime} \\
& +c\left(a_{1}, a_{2}\right) \int_{\mathbb{R}} d \xi \int_{\mathbb{R}^{2}}\left(|\tilde{f}|^{2}+\left|\nabla^{\prime} \tilde{g}\right|^{2}+\xi^{2}|\tilde{g}|^{2}\right)\left|x^{\prime}\right|^{-2 \mu} d x^{\prime} .
\end{aligned}
$$


Proof. In view of the definitions of $Q_{i}$ we have

$$
\begin{aligned}
& \int_{\mathbb{R}} d \xi \int_{\mathbb{R}^{2}} \xi^{2}|\tilde{v}|^{2}\left|x^{\prime}\right|^{-2 \mu-2} d x^{\prime}=\sum_{i=1}^{3} \int_{Q_{i}} \xi^{2}|\tilde{v}|^{2}\left|x^{\prime}\right|^{-2 \mu-2} d \xi d x^{\prime} \\
& \leq a_{1}^{2} \int_{Q_{1}}|\tilde{v}|^{2}\left|x^{\prime}\right|^{-2 \mu-4} d x^{\prime}+\frac{1}{a_{2}^{2}} \int_{Q_{2}} \xi^{4}|\tilde{v}|^{2}\left|x^{\prime}\right|^{-2 \mu} d x^{\prime}+\frac{1}{a_{1}^{2+2 \mu}} \int_{Q_{3}}|\tilde{v}|^{2} \xi^{4+2 \mu} d x^{\prime} .
\end{aligned}
$$

To examine the last term on the r.h.s. of (3.16) we introduce the sets

$$
\begin{aligned}
& d_{1}(\xi)=\left\{x^{\prime} \in \mathbb{R}^{2}:|\xi|\left|x^{\prime}\right| \leq a_{1}\right\}, \\
& d_{2}(\xi)=\left\{x^{\prime} \in \mathbb{R}^{2}:|\xi|\left|x^{\prime}\right| \geq a_{2}\right\}, \\
& d_{3}(\xi)=\left\{x^{\prime} \in \mathbb{R}^{2}: a_{1} \leq|\xi|\left|x^{\prime}\right| \leq a_{2}\right\},
\end{aligned}
$$

and for $\lambda>0$ we define

$$
\begin{aligned}
\Omega^{\lambda} & =\left\{\left(x^{\prime}, \xi\right) \in\left(\mathbb{R}^{2}\right) \times \mathbb{R}: \lambda|\xi|\left|x^{\prime}\right| \leq 1\right\}, \\
w^{\lambda}(\xi) & =\left\{x^{\prime} \in \mathbb{R}^{2}: \lambda|\xi|\left|x^{\prime}\right| \leq 1\right\} .
\end{aligned}
$$

Notice that $Q_{3} \subset \Omega^{\lambda}$ for $\lambda \in\left(0, a_{2}^{-1}\right]$. Next, we introduce a function $\chi \in$ $C^{\infty}\left(\mathbb{R}_{+}\right)$with the properties: $\chi(t)=1$ for $t \leq 1, \chi(t)=0$ for $t \geq 2$, $0 \leq \chi(t) \leq 1$ for $t \in \mathbb{R}_{+}$and $\chi^{\prime}(t) \leq 2$ for $t \in \mathbb{R}_{+}$.

Denote $\chi_{\lambda}\left(x^{\prime}, \xi\right)=\chi\left(\lambda|\xi|\left|x^{\prime}\right|\right)$. Then $\chi_{\lambda}\left(x^{\prime}, \xi\right)=1$ for $|\xi|\left|x^{\prime}\right| \leq \lambda^{-1}$ and $\chi_{\lambda}\left(x^{\prime}, \xi\right)=0|\xi|\left|x^{\prime}\right| \geq 2 \lambda^{-1}$.

Let $\tilde{\phi}$ be a solution of the equation

$$
\begin{gathered}
\operatorname{div}^{\prime}\left(\nabla^{\prime} \tilde{\phi}-\tilde{v}^{\prime} \chi_{\lambda}^{2}\right)-i \xi\left(-i \xi \tilde{\phi}-\tilde{v}_{3} \chi_{\lambda}^{2}\right)=0 \quad \text { in } \mathbb{R}^{3}, \\
\tilde{\phi} \rightarrow 0 \quad \text { as }\left|x^{\prime}\right| \rightarrow \infty .
\end{gathered}
$$

Multiplying $(2.2)_{1}$ by $\overline{\tilde{v}}^{\prime} \chi_{\lambda}^{2}-\nabla^{\prime} \overline{\tilde{\phi}},(2.3)$ by $\overline{\tilde{v}}_{3} \chi_{\lambda}^{2}+i \xi \overline{\tilde{\phi}}$, adding the results, integrating over $\mathbb{R}^{2}$ (by using integration by parts) and using (3.17) together with the assumption about the supports of $v$ and $p$ we obtain

$$
\begin{aligned}
\int_{\mathbb{R}^{2}} \nabla^{\prime} \tilde{v}^{\prime} \cdot \nabla^{\prime}\left(\overline{\tilde{v}}^{\prime} \chi_{\lambda}^{2}-\nabla^{\prime} \overline{\tilde{\phi}}\right) d x^{\prime}+\int_{\mathbb{R}^{2}} \xi^{2} \tilde{v}^{\prime}\left(\overline{\tilde{v}}^{\prime} \chi_{\lambda}^{2}-\nabla^{\prime} \overline{\tilde{\phi}}\right) d x^{\prime} \\
+\int_{\mathbb{R}^{2}} \nabla^{\prime} \tilde{v}_{3} \cdot \nabla^{\prime}\left(\overline{\tilde{v}}_{3} \chi_{\lambda}^{2}+\overline{i \xi \tilde{\phi}}\right) d x^{\prime}+\int_{\mathbb{R}^{2}} \xi^{2} \tilde{v}_{3}\left(\overline{\tilde{v}}_{3} \chi_{\lambda}^{2}-i \overline{\xi \xi \tilde{\phi}}\right) d x^{\prime} \\
=\int_{\mathbb{R}^{2}}\left[\tilde{f}^{\prime}\left(\overline{\tilde{v}}^{\prime} \chi_{\lambda}^{2}-\nabla^{\prime} \overline{\tilde{\phi}}\right)+\tilde{f}_{3}\left(\overline{\tilde{v}}_{3} \chi_{\lambda}^{2}+i \xi \tilde{\tilde{\phi}}\right)\right] d x^{\prime} .
\end{aligned}
$$

Hence by $(2.2)_{2}$ we have

$$
\begin{array}{r}
\int_{\mathbb{R}^{2}}\left(\left|\nabla^{\prime} \tilde{v}\right|^{2}+\xi^{2}|\tilde{v}|^{2}\right) \chi_{\lambda}^{2} d x^{\prime}=\int_{\mathbb{R}^{2}} \nabla^{\prime} \tilde{v} \tilde{\tilde{v}} \cdot 2 \nabla \chi_{\lambda} \chi_{\lambda} d x^{\prime}+\int_{\mathbb{R}^{2}}\left(\tilde{f}^{\prime} \cdot \overline{\tilde{v}}^{\prime}+\tilde{f}_{3} \overline{\tilde{v}}_{3}\right) \chi_{\lambda}^{2} d x^{\prime} \\
-\int_{\mathbb{R}^{2}}\left(\tilde{f}^{\prime} \cdot \nabla^{\prime} \overline{\tilde{\phi}}+\tilde{f}_{3} i \xi \overline{\tilde{\phi}}\right) d x^{\prime}+\int_{\mathbb{R}^{2}} \nabla^{\prime} \tilde{g} \cdot \nabla^{\prime} \tilde{\bar{\phi}} d x^{\prime}-\int_{\mathbb{R}^{2}} \xi^{2} \tilde{g} \tilde{\bar{\phi}} d x^{\prime} .
\end{array}
$$


Continuing, we get

$$
\begin{aligned}
\int_{\mathbb{R}^{2}}\left(\left|\nabla^{\prime} \tilde{v}\right|^{2}+\xi^{2}|\tilde{v}|^{2}\right) \chi_{\lambda}^{2} d x^{\prime} \leq \frac{\varepsilon_{1}}{2} \int_{\mathbb{R}^{2}}\left|\nabla^{\prime} \tilde{v}\right|^{2} \chi_{\lambda}^{2} d x^{\prime} \\
+\frac{2}{\varepsilon_{1}} \int_{\mathbb{R}^{2}}|\tilde{v}|^{2}\left|\nabla \chi_{\lambda}\right|^{2} d x^{\prime}+\frac{\varepsilon_{2}}{2} \int_{\mathbb{R}^{2}}|\xi|^{2+2 \mu}|\tilde{v}|^{2}\left|x^{\prime}\right|^{2 \mu} \chi_{\lambda}^{2} d x^{\prime} \\
+\frac{1}{2 \varepsilon_{2}} \frac{1}{|\xi|^{2+2 \mu}} \int_{\mathbb{R}^{2}}|\tilde{f}|^{2}\left|x^{\prime}\right|^{-2 \mu} \chi_{\lambda}^{2} d x^{\prime} \\
+\frac{\varepsilon_{3}}{2} \int_{\mathbb{R}^{2}}|\xi|^{2+2 \mu}\left(\left|\nabla^{\prime} \tilde{\phi}\right|^{2}+\xi^{2}|\tilde{\phi}|^{2}\right)\left|x^{\prime}\right|^{2 \mu} d x^{\prime} \\
+\frac{1}{2 \varepsilon_{3}} \frac{1}{|\xi|^{2+2 \mu}} \int_{\mathbb{R}^{2}}\left(|\tilde{f}|^{2}+\left|\nabla^{\prime} \tilde{g}\right|^{2}+\xi^{2}|\tilde{g}|^{2}\right)\left|x^{\prime}\right|^{-2 \mu} d x^{\prime}
\end{aligned}
$$

For $\left(x^{\prime}, \xi\right)$ such that $\lambda|\xi|\left|x^{\prime}\right| \in \operatorname{supp} \chi_{\lambda}$ we have

$$
\left|x^{\prime}\right|^{2 \mu}|\xi|^{2 \mu} \leq(2 / \lambda)^{2 \mu} \text {. }
$$

Therefore, taking $\varepsilon_{1}=1, \varepsilon_{2}=(\lambda / 2)^{2 \mu}$ in (3.18) and then multiplying (3.18) by $|\xi|^{2+2 \mu}$ and integrating with respect to $\xi$ yields

$$
\begin{aligned}
\frac{1}{2} \int_{\mathbb{R}} d \xi|\xi|^{2+2 \mu} & \int_{\mathbb{R}^{2}}\left(\left|\nabla^{\prime} \tilde{v}\right|^{2}+\xi^{2}|\tilde{v}|^{2}\right) \chi_{\lambda}^{2} d x^{\prime} \\
\leq & 2 \int_{\mathbb{R}} d \xi|\xi|^{2+2 \mu} \int_{\mathbb{R}^{2}}|\tilde{v}|^{2}\left|\nabla \chi_{\lambda}\right|^{2} d x^{\prime} \\
& +\frac{\varepsilon_{3}}{2} \int_{\mathbb{R}} d \xi|\xi|^{4+4 \mu} \int_{\mathbb{R}^{2}}\left(\left|\nabla^{\prime} \tilde{\phi}\right|^{2}+\xi^{2}|\tilde{\phi}|^{2}\right)\left|x^{\prime}\right|^{2 \mu} d x^{\prime} \\
& +c\left(\frac{1}{\lambda}\right) \int_{\mathbb{R}} d \xi \int_{\mathbb{R}^{2}}\left(|\tilde{f}|^{2}+\left|\nabla^{\prime} \tilde{g}\right|^{2}+\xi^{2}|\tilde{g}|^{2}\right)\left|x^{\prime}\right|^{-2 \mu} d x^{\prime}
\end{aligned}
$$

To examine the term

$$
\int_{\mathbb{R}} d \xi|\xi|^{4+4 \mu} \int_{\mathbb{R}^{2}}\left(\left|\nabla^{\prime} \tilde{\phi}\right|^{2}+\xi^{2}|\tilde{\phi}|^{2}\right)\left|x^{\prime}\right|^{2 \mu} d x^{\prime}=\int_{\mathbb{R}^{3}}\left|\partial_{x_{3}}^{2+2 \mu} \nabla \phi\right|^{2}\left|x^{\prime}\right|^{2 \mu} d x
$$

(where $\partial_{x_{3}}^{2+2 \mu}$ is the fractional derivative) we rewrite equation (3.17) in the form

$$
\Delta \phi=\operatorname{div}\left(v * F^{-1} \chi_{\lambda}^{2}\right) \quad \text { in } \mathbb{R}^{3},
$$

where $*$ denotes convolution with respect to $x_{3}$. Then $\partial_{x_{3}}^{2+2 \mu} \phi$ satisfies the equation

$$
\Delta \partial_{x_{3}}^{2+2 \mu} \phi=\operatorname{div} \partial_{x_{3}}^{2+2 \mu}\left(v * F^{-1} \chi_{\lambda}^{2}\right) .
$$


Therefore, by Lemma 2.3,

$$
\int_{\mathbb{R}^{3}}\left|\partial_{x_{3}}^{2+2 \mu} \nabla \phi\right|^{2}\left|x^{\prime}\right|^{2 \mu} d x \leq c_{1} \int_{\mathbb{R}^{3}}\left|\partial_{x_{3}}^{2+2 \mu} v * F^{-1} \chi_{\lambda}^{2}\right|^{2}\left|x^{\prime}\right|^{2 \mu} d x,
$$

and the Parserval identity together with (3.19) implies

$$
\begin{aligned}
\int_{\mathbb{R}} d \xi \int_{\mathbb{R}^{2}}|\xi|^{4+4 \mu}\left(\left|\nabla^{\prime} \tilde{\phi}\right|^{2}+\xi^{2}|\tilde{\phi}|^{2}\right)\left|x^{\prime}\right|^{2 \mu} d x^{\prime} & \leq c_{1} \int_{\mathbb{R}} d \xi \int_{\mathbb{R}^{2}}|\xi|^{4+4 \mu}|\tilde{v}|^{2} \chi_{\lambda}^{4}\left|x^{\prime}\right|^{2 \mu} d x^{\prime} \\
& \leq c_{1}\left(\frac{2}{\lambda}\right)^{2 \mu} \int_{\mathbb{R}} d \xi \int_{\mathbb{R}^{2}}|\xi|^{4+2 \mu}|\tilde{v}|^{2} \chi_{\lambda}^{2} d x^{\prime}
\end{aligned}
$$

where we also used the inequality $\chi_{\lambda}^{2} \leq 1$.

Assuming that $\left(\varepsilon_{3} / 2\right) c_{1}(2 / \lambda)^{2 \mu}=1 / 4$, inequality (3.20) gives

$$
\begin{aligned}
\frac{1}{4} \int_{\mathbb{R}} d \xi|\xi|^{2+2 \mu} & \int_{\mathbb{R}^{2}}\left(\left|\nabla^{\prime} \tilde{v}\right|^{2}+\xi^{2}|\tilde{v}|^{2}\right) \chi_{\lambda}^{2} d x^{\prime} \\
\leq & 2 \int_{\mathbb{R}} d \xi|\xi|^{2+2 \mu} \int_{\mathbb{R}^{2}}|\tilde{v}|^{2}\left|\nabla \chi_{\lambda}\right|^{2} d x^{\prime} \\
& +c\left(\frac{1}{\lambda}\right) \int_{\mathbb{R}} d \xi \int_{\mathbb{R}^{2}}\left(|\tilde{f}|^{2}+\left|\nabla^{\prime} \tilde{g}\right|^{2}+\xi^{2}|\tilde{g}|^{2}\right)\left|x^{\prime}\right|^{-2 \mu} d x^{\prime} .
\end{aligned}
$$

Using in (3.21) the inequality $\left|\nabla \chi_{\lambda}\right| \leq 2 \lambda|\xi|$ and assuming that $\lambda \leq 2$ we obtain

$$
\begin{aligned}
\int_{\mathbb{R}} d \xi|\xi|^{2+2 \mu} \int_{w^{\lambda}(\xi)} \xi^{2}|\tilde{v}|^{2} d x^{\prime} \leq & 32 \lambda^{2} \int_{\mathbb{R}} d \xi|\xi|^{2+2 \mu} \int_{w^{\lambda / 2}(\xi) \backslash w^{\lambda}(\xi)} \xi^{2}|\tilde{v}|^{2} d x^{\prime} \\
& +c\left(\frac{1}{\lambda}\right) \int_{\mathbb{R}} d \xi \int_{\mathbb{R}^{2}}\left(|\tilde{f}|^{2}+\left|\nabla^{\prime} \tilde{g}\right|^{2}+\xi^{2}|\tilde{g}|^{2}\right)\left|x^{\prime}\right|^{-2 \mu} d x^{\prime} .
\end{aligned}
$$

Multiplying by $(\lambda / 2)^{2 \mu+2}$ we get

$$
\begin{aligned}
\left(\frac{\lambda}{2}\right)^{2 \mu+2} & \int_{\mathbb{R}} d \xi|\xi|^{2+2 \mu} \int_{w^{\lambda}(\xi)} \xi^{2}|\tilde{v}|^{2} d x^{\prime} \\
\leq & 2 \cdot 4^{3+\mu} \lambda^{2}\left(\frac{\lambda / 2}{2}\right)^{2 \mu+2} \int_{\mathbb{R}} d \xi|\xi|^{2+2 \mu} \int_{w^{\lambda / 2}(\xi) \backslash w^{\lambda}(\xi)} \xi^{2}|\tilde{v}|^{2} d x^{\prime} \\
& +c(\lambda) \int_{\mathbb{R}} d \xi \int_{\mathbb{R}^{2}}\left(|\tilde{f}|^{2}+\left|\nabla^{\prime} \tilde{g}\right|^{2}+\xi^{2}|\tilde{g}|^{2}\right)\left|x^{\prime}\right|^{-2 \mu} d x^{\prime} .
\end{aligned}
$$

Let $2 \cdot 4^{3+\mu} \lambda^{2} \leq 1 / 2$. Then iterating (3.22) $k$ times we obtain 


$$
\begin{aligned}
\left(\frac{\lambda}{2}\right)^{2 \mu+2} & \int_{\mathbb{R}} d \xi|\xi|^{2+2 \mu} \int_{w^{\lambda}(\xi)} \xi^{2}|\tilde{v}|^{2} d x^{\prime} \\
\leq & \frac{1}{2^{k}}\left(\frac{\lambda / 2^{k}}{2}\right)^{2 \mu+2} \int_{\mathbb{R}} d \xi|\xi|^{2+2 \mu} \int_{w^{\lambda / 2^{k+1}}(\xi) \backslash w^{\lambda / 2^{k}}(\xi)} \xi^{2}|\tilde{v}|^{2} d x^{\prime} \\
& +c\left(k, \frac{1}{\lambda}\right) \int_{\mathbb{R}} d \xi \int_{\mathbb{R}^{2}}\left(|\tilde{f}|^{2}+\left|\nabla^{\prime} \tilde{g}\right|^{2}+\xi^{2}|\tilde{g}|^{2}\right)\left|x^{\prime}\right|^{-2 \mu} d x^{\prime}
\end{aligned}
$$

where

$$
w^{\lambda / 2^{k+1}}(\xi) \backslash w^{\lambda / 2^{k}}(\xi)=\left\{x^{\prime} \in \mathbb{R}^{2}: \frac{1}{\lambda / 2^{k}} \leq\left|x^{\prime}\right||\xi| \leq \frac{1}{\lambda / 2^{k+1}}\right\} .
$$

Since $|\xi| \leq\left(2^{k+1} / \lambda\right)\left|x^{\prime}\right|^{-1}$ for $\left(x^{\prime}, \xi\right)$ such that $x^{\prime} \in w^{\lambda / 2^{k+1}}(\xi) \backslash w^{\lambda / 2^{k}}(\xi)$, the first term on the r.h.s. of (3.23) is estimated by

$$
\begin{aligned}
\frac{1}{2^{k}}\left(\frac{\lambda}{2^{k+1}}\right)^{2 \mu+2} \int_{\mathbb{R}} d \xi \xi^{2} & \int_{w^{\lambda / 2^{k+1}}(\xi) \backslash w^{\lambda / 2^{k}}(\xi)}|\tilde{v}|^{2}\left(\frac{2^{k+1}}{\lambda}\right)^{2 \mu+2}\left|x^{\prime}\right|^{-2 \mu-2} d x^{\prime} \\
& =\frac{1}{2^{k}} \int_{\mathbb{R}} d \xi \xi^{2} \int_{w^{\lambda / 2^{k+1}}(\xi) \backslash w^{\lambda / 2^{k}}(\xi)}|\tilde{v}|^{2}\left|x^{\prime}\right|^{-2 \mu-2} d x^{\prime} \\
\leq & \frac{1}{2^{k}} \int_{\mathbb{R}} d \xi \xi^{2} \int_{\mathbb{R}^{2}}|\tilde{v}|^{2}\left|x^{\prime}\right|^{-2 \mu-2} d x^{\prime}
\end{aligned}
$$

Hence

$$
\begin{array}{r}
\int_{\mathbb{R}} d \xi|\xi|^{2+2 \mu} \int_{w^{\lambda}(\xi)} \xi^{2}|\tilde{v}|^{2} d x^{\prime} \leq \frac{1}{2^{k}}\left(\frac{2}{\lambda}\right)^{2 \mu+2} \int_{\mathbb{R}} d \xi \xi^{2} \int_{\mathbb{R}^{2}}|\tilde{v}|^{2}\left|x^{\prime}\right|^{-2 \mu-2} d x \\
+c(\lambda, k) \int_{\mathbb{R}} d \xi \int_{\mathbb{R}^{2}}\left(|\tilde{f}|^{2}+\left|\nabla^{\prime} \tilde{g}\right|^{2}+\xi^{2}|\tilde{g}|^{2}\right)\left|x^{\prime}\right|^{-2 \mu} d x^{\prime}
\end{array}
$$

Let $k$ be so large that $k>2+2 \mu$ and $1 /\left(2^{k-2-3 \mu} \lambda^{2 \mu+2} a_{1}^{2+2 \mu}\right) \leq 1 / 2$. Then by (3.16) and (3.24) inequality (3.15) follows.

As a consequence of Lemmas 3.2-3.4 we can formulate

Corollary 3.5. Let $\mu \in(0,1)$ and let $(v, p) \in H_{-\mu}^{2}\left(\mathbb{R}^{3}\right) \times H_{-\mu}^{2}\left(\mathbb{R}^{3}\right)$ be such that supp $v \subset\left(\mathbb{R}^{3} \backslash \bar{C}_{\delta_{0}}\right) \cap C_{R, a}$, supp $p \subset\left(\mathbb{R}^{3} \backslash \bar{C}_{\delta_{0}}\right) \cap C_{R, a}$ be a solution to problem (2.1) with $f \in L_{2,-\mu}\left(\mathbb{R}^{3}\right), g \in H_{-\mu}^{1}\left(\mathbb{R}^{3}\right)$, supp $f \subset\left(\mathbb{R}^{3} \backslash \bar{C}_{\delta_{0}}\right) \cap C_{R, a}$, $\operatorname{supp} g \subset\left(\mathbb{R}^{3} \backslash \bar{C}_{\delta_{0}}\right) \cap C_{R, a}$. Then

$$
\|v\|_{H_{-\mu}^{2}\left(\mathbb{R}^{3}\right)}+\|p\|_{H_{-\mu}^{1}\left(\mathbb{R}^{3}\right)} \leq c\left(\|f\|_{L_{2,-\mu}\left(\mathbb{R}^{3}\right)}+\|g\|_{H_{-\mu}^{1}\left(\mathbb{R}^{3}\right)}\right) .
$$

Proof. Using (3.15) in (3.4) and assuming that $a_{1}$ is sufficiently small and $a_{2}$ is sufficiently large, we obtain inequality (3.25). This ends the proof. 
Proof of Theorem 3.1. Estimate (3.1) holds by Lemma 2.4 and Corollary 3.5 .

4. Existence of a solution to problem (1.1). Let $C_{\delta}=\left\{x \in \mathbb{R}^{3}\right.$ : $\left.0<\left|x^{\prime}\right|<\delta\right\}, \delta \leq \delta_{0}<R / 2, R>0$. Let $\mu \in(0,1), f \in L_{2,-\mu}(\Omega)$. Then we introduce the functions $f_{\delta}=f$ in $\Omega \backslash C_{\delta}$. Let $g \in V$. Then there exists a sequence $\bar{g}_{\delta} \in H^{1}(\Omega)$ with $\bar{g}_{\delta}=0$ in $C_{\delta}$ and $\int_{\Omega} \bar{g}_{\delta} d x=0$ such that

$$
\bar{g}_{\delta} \rightarrow g \quad \text { in } H_{-\mu}^{1}(\Omega), \quad \text { where } \quad \bar{g}_{\delta}= \begin{cases}g_{\delta} & \text { in } \Omega \backslash \bar{C}_{\delta}, \\ 0 & \text { in } C_{\delta} .\end{cases}
$$

Now, we consider the auxiliary problem

$$
\begin{array}{ll}
-\Delta v_{\delta}+\nabla p_{\delta}=f_{\delta} & \text { in } \Omega_{*} \backslash \bar{C}_{\delta}, \\
\operatorname{div} v_{\delta}=g_{\delta} & \text { in } \Omega_{*} \backslash \bar{C}_{\delta}, \\
v_{\delta}=0 & \text { on }\left\{x \in \Omega_{*}:\right. \\
\bar{n} \cdot \mathbb{D}(v) \cdot \bar{\tau}_{\alpha}=0, \quad v \cdot \bar{n}=0, \quad \alpha=1,2, & \text { on } \partial \Omega_{*} \backslash \bar{C}_{\delta} .
\end{array}
$$

In view of the assumptions on $\Omega$ (see (1.3)-(1.4)) there exists a unique solution $v_{\delta} \in H^{2}\left(\Omega \backslash \bar{C}_{\delta}\right), p_{\delta} \in H^{1}\left(\Omega \backslash \bar{C}_{\delta}\right)$ with $\int_{\Omega \backslash \bar{C}_{\delta}} p_{\delta} d x=0$ of problem (4.2).

LEMma 4.1. For the solution $\left(v_{\delta}, p_{\delta}\right)$ satisfying the condition

$$
\int_{\Omega \backslash \bar{C}_{\delta}} p_{\delta} d x=0
$$

we have the estimate

$$
\left\|p_{\delta}\right\|_{L_{2}\left(\Omega \backslash \bar{C}_{\delta}\right)}+\left\|v_{\delta}\right\|_{H^{1}\left(\Omega \backslash \bar{C}_{\delta}\right)} \leq c\left(\left\|f_{\delta}\right\|_{L_{2}\left(\Omega \backslash \bar{C}_{\delta}\right)}+\left\|g_{\delta}\right\|_{H^{1}\left(\Omega \backslash \bar{C}_{\delta}\right)}\right)
$$

where $c>0$ does not depend on $\delta$.

Proof. First, notice that $\left(v_{\delta}, p_{\delta}\right)$ satisfies the identity

$$
\int_{\Omega \backslash \bar{C}_{\delta}} \mathbb{D} v_{\delta}: \mathbb{D} \psi d x+\int_{\Omega \backslash \bar{C}_{\delta}} p_{\delta} \operatorname{div} \psi d x=\int_{\Omega \backslash \bar{C}_{\delta}} f_{\delta} \cdot \psi d x-\int_{\Omega \backslash \bar{C}_{\delta}} \nabla g_{\delta} \cdot \psi d x
$$

for every $\psi \in H^{1}\left(\Omega \backslash \bar{C}_{\delta}\right)$ with $\psi=0$ on $\left\{x \in \Omega:\left|x^{\prime}\right|=\delta\right\}$. Inserting $\psi=v_{\delta}$ in (4.5) and using the Korn inequality we get

$$
\begin{aligned}
\left\|v_{\delta}\right\|_{H^{1}\left(\Omega \backslash \bar{C}_{\delta}\right)}^{2} \leq & \varepsilon_{1}\left(\left\|v_{\delta}\right\|_{L_{2}\left(\Omega \backslash \bar{C}_{\delta}\right)}^{2}+\left\|p_{\delta}\right\|_{L_{2}\left(\Omega \backslash \bar{C}_{\delta}\right)}^{2}\right) \\
& +c\left(1 / \varepsilon_{1}\right)\left(\left\|f_{\delta}\right\|_{L_{2}\left(\Omega \backslash \bar{C}_{\delta}\right)}^{2}+\left\|g_{\delta}\right\|_{H^{1}\left(\Omega \backslash \bar{C}_{\delta}\right)}^{2}\right) .
\end{aligned}
$$

Now, let $\phi$ be a solution of the problem

$$
\begin{aligned}
& \operatorname{div} \phi=p_{\delta} \quad \text { in } \Omega_{*} \backslash \bar{C}_{\delta}, \\
& \left.\phi\right|_{\partial\left(\Omega_{*} \backslash \bar{C}_{\delta}\right)}=0 .
\end{aligned}
$$


By [3], $\phi$ satisfies

$$
\|\phi\|_{H^{1}\left(\Omega \backslash \bar{C}_{\delta}\right)} \leq c\left\|p_{\delta}\right\|_{L_{2}\left(\Omega \backslash \bar{C}_{\delta}\right)},
$$

where $c>0$ does not depend on $\delta$. Inserting $\psi=\phi$ to (4.5) we get

$$
\begin{aligned}
\left\|p_{\delta}\right\|_{L_{2}\left(\Omega \backslash \bar{C}_{\delta}\right)}^{2} \leq & \varepsilon_{2}\|\phi\|_{H^{1}\left(\Omega \backslash \bar{C}_{\delta}\right)}^{2}+c\left(\frac{1}{\varepsilon_{2}}\right)\left(\left\|f_{\delta}\right\|_{L_{2}\left(\Omega \backslash \bar{C}_{\delta}\right)}^{2}\right. \\
& \left.+\left\|g_{\delta}\right\|_{H^{1}\left(\Omega \backslash \bar{C}_{\delta}\right)}\left\|\nabla v_{\delta}\right\|_{L_{2}\left(\Omega \backslash \bar{C}_{\delta}\right)}^{2}\right) .
\end{aligned}
$$

Therefore, assuming that $\varepsilon_{1}, \varepsilon_{2}$ are sufficiently small, by (4.6)-(4.8) and the Poincaré inequality, the estimate (4.4) follows.

Let $\mu \in(0,1)$. Since $\left(v_{\delta}, p_{\delta}\right) \in H^{2}\left(\Omega \backslash \bar{C}_{\delta}\right) \times H^{1}\left(\Omega \backslash \bar{C}_{\delta}\right)$, we have $\left(v_{\delta}, p_{\delta}\right) \in$ $H_{-\mu}^{2}\left(\Omega \backslash \bar{C}_{\delta}\right) \times H_{-\mu}^{1}\left(\Omega \backslash \bar{C}_{\delta}\right)$. Now, our aim is to prove that for some solution $\left(v_{\delta}, p_{\delta}\right)$ of $(4.2)$, where $p_{\delta}$ may differ by a constant from $p_{\delta}$ satisfying (4.3),

$$
\left\|v_{\delta}\right\|_{H_{-\mu}^{2}\left(\Omega \backslash \bar{C}_{\delta}\right)}+\left\|p_{\delta}\right\|_{H_{-\mu}^{1}\left(\Omega \backslash \bar{C}_{\delta}\right)} \leq c\left(\left\|f_{\delta}\right\|_{L_{2,-\mu}\left(\Omega \backslash \bar{C}_{\delta}\right)}+\left\|g_{\delta}\right\|_{H_{-\mu}^{1}\left(\Omega \backslash \bar{C}_{\delta}\right)}\right),
$$

where $c>0$ is a constant independent of $\delta$.

To do this we need to localize problem (4.2) in neighbourhoods of four types of points:

(a) near an interior point of $L$;

(b) near a point where $L$ meets $\partial \Omega_{*}$;

(c) near an interior point of $\Omega$ but at a positive distance from $L$;

(d) near a point of $\partial \Omega$ at a positive distance from $L$.

Localization of problem (4.2) near an interior point of $L$. Let $x^{0}=\left(0,0, x_{3}^{0}\right)$ be an arbitrary point of $L \cap \Omega_{*}$ and let $\zeta \in C_{0}^{\infty}(\Omega)$ be a function with the properties: $0 \leq \zeta(x) \leq 1$ for $x \in \Omega_{*} ; \operatorname{supp} \zeta \subset \tilde{\Omega} \equiv C_{R, a}=$ $\left\{x:\left|x^{\prime}\right|<R,\left|x_{3}-x_{3}^{0}\right|<a\right\}$, where $R>0, a>0$ are such that $\bar{C}_{R, a} \subset \Omega_{*}$; $\zeta(x)=1$ for $x \in C_{R / 2, a / 2} ;\left|D^{\alpha} \zeta(x)\right| \leq c_{\alpha} \gamma^{-|\alpha|}, \gamma=\min (R, a)$. Then the function $\left(\tilde{v}_{\delta}, \tilde{p}_{\delta}\right)=\left(v_{\delta} \zeta, p_{\delta} \zeta\right)$ is a solution of the problem

$$
\begin{array}{ll}
-\Delta \tilde{v}_{\delta}+\nabla \tilde{p}_{\delta}=\tilde{f}_{\delta}-2 \nabla v_{\delta} \nabla \zeta-v_{\delta} \Delta \zeta+p_{\delta} \nabla \zeta \equiv \tilde{F}_{\delta} \\
\operatorname{div} \tilde{v}_{\delta}=\tilde{g}_{\delta}+v_{\delta} \cdot \nabla \zeta \equiv \tilde{G}_{\delta} & \text { in } \tilde{\Omega} \cap\left(\Omega_{*} \backslash \bar{C}_{\delta}\right), \\
\tilde{v}_{\delta}=0 & \text { in } \tilde{\Omega} \cap\left(\Omega_{*} \backslash \bar{C}_{\delta}\right),
\end{array}
$$

where $\tilde{f}_{\delta}=f_{\delta} \zeta, \tilde{g}_{\delta}=g_{\delta} \zeta$.

LEMmA 4.2. Let $\mu \in(0,1), \delta \leq \delta_{0}<R / 2, f_{\delta} \in L_{2,-\mu}\left(\Omega \backslash \bar{C}_{\delta}\right), g_{\delta} \in$ $H_{-\mu}^{1}\left(\Omega \backslash \bar{C}_{\delta}\right), g_{\delta}=0$ on $\partial\left(\Omega \backslash \bar{C}_{\delta}\right), \int_{\Omega \backslash \bar{C}_{\delta}} g_{\delta} d x=0$. Then the solution 
$\left(\tilde{v}_{\delta}, \tilde{p}_{\delta}\right)$ of problem (4.10) satisfies the estimate

$$
\begin{aligned}
& \left\|\tilde{v}_{\delta}\right\|_{H_{-\mu}^{2}\left(\tilde{\Omega} \cap\left(\Omega \backslash \bar{C}_{\delta}\right)\right)}+\left\|\tilde{p}_{\delta}\right\|_{H_{-\mu}^{1}\left(\tilde{\Omega} \cap\left(\Omega \backslash \bar{C}_{\delta}\right)\right)} \\
& \leq c \frac{\delta_{0}}{\gamma}\left(\left\|p_{\delta}\right\|_{L_{2,-\mu-1}\left(\Omega \backslash \bar{C}_{\delta}\right)}+\left\|\nabla v_{\delta}\right\|_{L_{2,-\mu-1}\left(\Omega \backslash \bar{C}_{\delta}\right)}+\left\|v_{\delta}\right\|_{L_{2,-\mu-2}\left(\Omega \backslash \bar{C}_{\delta}\right)}\right) \\
& \quad+c\left(\left\|f_{\delta}\right\|_{L_{2,-\mu}\left(\Omega \backslash \bar{C}_{\delta}\right)}+\left\|g_{\delta}\right\|_{H_{-\mu}^{1}\left(\Omega \backslash \bar{C}_{\delta}\right)}\right)
\end{aligned}
$$

where $c>0$ is independent of $\delta$.

Proof. Let us extend the functions $\tilde{v}_{\delta}, \tilde{p}_{\delta}, \tilde{F}_{\delta}, \tilde{g}_{\delta}$ by zero on $\mathbb{R}^{3} \backslash\left(C_{R, a} \backslash \bar{C}_{\delta}\right)$ to functions still denoted by $\tilde{v}_{\delta}, \tilde{p}_{\delta}, \tilde{F}_{\delta}, \tilde{g}_{\delta}$ such that $\tilde{v}_{\delta}$ and $\tilde{p}_{\delta}$ satisfy the problem

$$
\begin{array}{ll}
-\Delta \tilde{v}_{\delta}+\nabla \tilde{p}_{\delta}=\tilde{F}_{\delta} & \text { in } \mathbb{R}^{3} \backslash \bar{C}_{\delta}, \\
\operatorname{div} \tilde{v}_{\delta}=\tilde{G}_{\delta} & \text { in } \mathbb{R}^{3} \backslash \bar{C}_{\delta}, \\
\tilde{v}_{\delta}=0 & \text { on } \partial C_{\delta}, \\
\tilde{v}_{\delta} \rightarrow 0 & \text { as }|x| \rightarrow \infty,
\end{array}
$$

where $C_{\delta}=\left\{x \in \mathbb{R}^{3}: 0<\left|x^{\prime}\right|<\delta\right\}$. Since $\left(\tilde{v}_{\delta}, \tilde{p}_{\delta}\right) \in H_{-\mu}^{2}\left(\mathbb{R}^{3} \backslash \bar{C}_{\delta}\right) \times$ $H_{-\mu}^{1}\left(\mathbb{R}^{3} \backslash \bar{C}_{\delta}\right)$, by Theorem 3.1 we have the estimate

$$
\begin{aligned}
& \left\|\tilde{v}_{\delta}\right\|_{H_{-\mu}^{2}\left(\mathbb{R}^{3} \backslash \bar{C}_{\delta}\right)}+\left\|\tilde{p}_{\delta}\right\|_{H_{-\mu}^{1}\left(\mathbb{R}^{3} \backslash \bar{C}_{\delta}\right)} \\
\leq & c\left(\left\|\tilde{F}_{\delta}\right\|_{L_{2,-\mu}\left(\mathbb{R}^{3} \backslash \bar{C}_{\delta}\right)}+\left\|\tilde{G}_{\delta}\right\|_{H_{-\mu}^{1}\left(\mathbb{R}^{3} \backslash \bar{C}_{\delta}\right)}\right) \\
\leq & c\left(\left\|\tilde{f}_{\delta}\right\|_{L_{2,-\mu}\left(\mathbb{R}^{3} \backslash \bar{C}_{\delta}\right)}+\left\|\tilde{g}_{\delta}\right\|_{H_{-\mu}^{1}\left(\mathbb{R}^{3} \backslash \bar{C}_{\delta}\right)}+\left\|\nabla v_{\delta} \nabla \zeta\right\|_{L_{2,-\mu}\left(\Omega \backslash \bar{C}_{\delta}\right)}\right. \\
& \left.+\left\|v_{\delta} \Delta \zeta\right\|_{L_{2,-\mu}\left(\Omega \backslash \bar{C}_{\delta}\right)}+\left\|p_{\delta} \nabla \zeta\right\|_{L_{2,-\mu}\left(\Omega \backslash \bar{C}_{\delta}\right)}+\left\|v_{\delta} \nabla \zeta\right\|_{L_{2,-\mu-1}\left(\Omega \backslash \bar{C}_{\delta}\right)}\right) .
\end{aligned}
$$

Consider the third term on the r.h.s. of (4.13). We have

$$
\begin{aligned}
& \left\|\nabla v_{\delta} \nabla \zeta\right\|_{L_{2,-\mu}\left(\Omega \backslash \bar{C}_{\delta}\right)}=\left\|\nabla v_{\delta} \nabla \zeta\right\|_{L_{2,-\mu}\left(\operatorname{supp} \nabla \zeta \cap\left(\Omega \backslash \bar{C}_{\delta}\right)\right)} \\
& \quad \leq\left\|\nabla v_{\delta} \nabla \zeta\right\|_{L_{2,-\mu}\left(\operatorname{supp} \nabla \zeta \cap\left(\Omega \backslash \bar{C}_{\delta_{0}}\right)\right)}+\left\|\nabla v_{\delta} \nabla \zeta\right\|_{L_{2,-\mu}\left(\operatorname{supp} \nabla \zeta \cap\left(C_{\delta_{0}} \backslash \bar{C}_{\delta}\right)\right)}
\end{aligned}
$$

First, using Lemma 4.1 we get

$$
\begin{aligned}
\left\|\nabla v_{\sigma} \nabla \zeta\right\|_{L_{2,-\mu}\left(\operatorname{supp} \nabla \zeta \cap\left(\Omega \backslash \bar{C}_{\delta_{0}}\right)\right)} & \leq c\left\|\nabla v_{\delta}\right\|_{L_{2}\left(\Omega \backslash \bar{C}_{\delta}\right)} \\
& \leq c\left(\left\|f_{\delta}\right\|_{L_{2}\left(\Omega \backslash \bar{C}_{\delta}\right)}+\left\|g_{\delta}\right\|_{L_{2}\left(\Omega \backslash \bar{C}_{\delta}\right)}\right) .
\end{aligned}
$$

Next, we obtain

$$
\left\|\nabla v_{\delta} \nabla \zeta\right\|_{L_{2,-\mu}\left(\operatorname{supp} \nabla \zeta \cap\left(C_{\delta_{0}} \backslash \bar{C}_{\delta}\right)\right)} \leq c \frac{\delta_{0}}{\gamma}\left\|\nabla v_{\delta}\right\|_{L_{2,-\mu-1}\left(\Omega \backslash \bar{C}_{\delta}\right)} .
$$

Hence

$$
\begin{aligned}
\left\|\nabla v_{\delta} \nabla \zeta\right\|_{L_{2,-\mu}\left(\Omega \backslash \bar{C}_{\delta}\right)} \leq & c \frac{\delta_{0}}{\gamma}\left\|\nabla v_{\delta}\right\|_{L_{2,-\mu-1}\left(\Omega \backslash \bar{C}_{\delta}\right)} \\
& +c\left(\left\|f_{\delta}\right\|_{L_{2,-\mu}\left(\Omega \backslash \bar{C}_{\delta}\right)}+\left\|g_{\delta}\right\|_{L_{2,-\mu}\left(\Omega \backslash \bar{C}_{\delta}\right)}\right) .
\end{aligned}
$$


The terms $\left\|v_{\delta} \Delta \zeta\right\|_{L_{2,-\mu}\left(\Omega \backslash \bar{C}_{\delta}\right)},\left\|v_{\delta} \nabla \zeta\right\|_{L_{2}\left(\Omega \backslash \bar{C}_{\delta}\right)}$ and $\left\|p_{\delta} \nabla \zeta\right\|_{L_{2,-\mu}\left(\Omega \backslash \bar{C}_{\delta}\right)}$ are estimated in the same way.

Taking into account the above estimates and inequality (4.13) we obtain (4.11). This ends the proof.

Localization of problem (4.2) near a point where $L$ intersects $\partial \Omega_{*}$. Let $L \cap \partial \Omega_{*}=\left\{x^{(1)}, x^{(2)}\right\}$. We localize problem (4.2) in neighbourhoods of the points $x^{(1)}$ and $x^{(2)}$. Consider for example $x^{(1)}$ and let $x^{(1)} \in$ $\tilde{\Omega} \cap \partial \Omega_{*}$, where $\tilde{\Omega}=C_{R, a}=\left\{x \in \mathbb{R}^{3}:\left|x^{\prime}\right|<R,\left|x_{3}-x_{3}^{(1)}\right|<a\right\}$, and $\operatorname{diam} \tilde{\Omega}=\lambda$. Next, introduce a function $\zeta \in C_{0}^{\infty}(\tilde{\Omega})$ such that $0 \leq \zeta(x) \leq 1$ for $x \in \tilde{\Omega}, \zeta(x)=1$ for $x \in \tilde{w} \equiv C_{R / 2, a / 2}$. Since $v_{\delta}=0$ on $\partial \Omega_{*}$, the functions $\tilde{v}_{\delta}=v \zeta$ and $\tilde{p}_{\delta}=p_{\delta} \zeta$ satisfy in $\tilde{\Omega} \cap\left(\Omega_{*} \backslash \bar{C}_{\delta}\right)$ the same problem as in the previous case, i.e. problem (4.10).

Lemma 4.3. Let the assumptions of Lemma 4.2 be satisfied. Then the solution $\left(\tilde{v}_{\delta}, \tilde{p}_{\delta}\right)$ of problem (4.10) in $\tilde{\Omega} \cap\left(\Omega_{*} \backslash \bar{C}_{\delta}\right)$ satisfies estimate (4.11).

Proof. By conditions (1.3)-(1.4) we can assume that the neighbourhood $\tilde{\Omega} \cap \partial \Omega_{*}$ of $x^{(1)}$ is flat, i.e. we can introduce a local Cartesian coordinate system $y=\left(y_{1}, y_{2}, y_{3}\right)$ with origin at $x^{(1)}$ such that the $y_{3}$ axis is directed opposite to the outward vector normal to $\partial \Omega_{*}$ and $\tilde{\Omega} \cap \partial \Omega_{*}$ lies in the plane $y_{3}=0$. Problem (4.10) is described in some coordinates $x=\left(x_{1}, x_{2}, x_{3}\right)$. Thus, passing to the coordinates $y$ can be made by a rotation and translation. Denote this mapping by $y=Y(x)$. Then extending appropriately by zero the functions $\tilde{v}_{\delta}, \tilde{p}_{\delta}, \tilde{F}_{\delta}, \tilde{g}_{\delta}$ we see that problem (4.10) takes the form

$$
\begin{array}{ll}
-\Delta \hat{v}_{\delta}+\nabla \hat{p}_{\delta}=\hat{F}_{\delta} & \text { in } \mathbb{R}_{+}^{3} \backslash \bar{C}_{+\delta}, \\
\operatorname{div} \hat{v}_{\delta}=\hat{G}_{\delta} & \text { in } \mathbb{R}_{+}^{3} \backslash \bar{C}_{+\delta}, \\
\hat{v}_{\delta}=0 & \text { on }\left\{y \in \mathbb{R}^{3}:\left|y^{\prime}\right|=\delta\right\}, \\
\frac{\partial \hat{v}_{\delta i}}{\partial y_{3}}=0, \quad i=1,2, \quad \hat{v}_{\delta 3}=0 & \text { on }\left\{y \in \mathbb{R}^{3}: y_{3}=0\right\} \backslash\left\{y \in \mathbb{R}^{3}:\left|y^{\prime}\right|<\delta\right\},
\end{array}
$$

where $\mathbb{R}_{+}^{3}=\left\{y \in \mathbb{R}^{3}: y_{3}>0\right\}, C_{+\delta}=\left\{y \in \mathbb{R}_{+}^{3}:\left|y^{\prime}\right|<\delta\right\}, \hat{u}(y)=$ $u\left(Y^{-1}(y)\right), u \in\left\{\tilde{v}_{\delta}, \tilde{p}_{\delta}, \tilde{F}_{\delta}, \tilde{G}_{\delta}\right\}$.

Now, set

$$
\begin{gathered}
W\left(y^{\prime}, y_{3}\right)= \begin{cases}w\left(y^{\prime},-y_{3}\right) & \text { for } y_{3}<0, \\
w\left(y^{\prime}, y_{3}\right) & \text { for } y_{3}>0,\end{cases} \\
U\left(y^{\prime}, y_{3}\right)= \begin{cases}-u\left(y^{\prime},-y_{3}\right) & \text { for } y_{3}<0 \\
u\left(y^{\prime}, y_{3}\right) & \text { for } y_{3}>0\end{cases}
\end{gathered}
$$

where $w \in\left\{\hat{v}_{\delta 1}, \hat{v}_{\delta 2}, \hat{p}_{\delta}, \hat{g}_{\delta}, \hat{F}_{\delta 1}, \hat{F}_{\delta 2}\right\}, W \in\left\{V_{\delta 1}, V_{\delta 2}, P_{\delta}, K_{\delta}, H_{\delta 1}, H_{\delta 2}\right\}, u \in$ $\left\{\hat{v}_{\delta 3}, \hat{F}_{\delta 3}\right\}, U \in\left\{V_{\delta 3}, H_{\delta 3}\right\}$. Then $\left(V_{\delta}, P_{\delta}\right) \in H_{-\mu}^{2}\left(\mathbb{R}^{3} \backslash \bar{C}_{\delta}\right) \times H_{-\mu}^{1}\left(\mathbb{R}^{3} \backslash \bar{C}_{\delta}\right)$ 
satisfies the problem

$$
\begin{array}{ll}
-\Delta V_{\delta}+\nabla P_{\delta}=H_{\delta} & \text { in } \mathbb{R}^{3} \backslash \bar{C}_{\delta}, \\
\operatorname{div} V_{\delta}=K_{\delta} & \text { in } \mathbb{R}^{3} \backslash \bar{C}_{\delta}, \\
V_{\delta}=0 & \text { on } \partial C_{\delta}, \\
V_{\delta} \rightarrow 0 & \text { as }|x| \rightarrow \infty
\end{array}
$$

Using Theorem 3.1 and repeating the argument from the proof of Lemma 4.2 we obtain

$$
\begin{aligned}
& \left\|\tilde{v}_{\delta}\right\|_{H_{-\mu}^{2}\left(\tilde{\Omega} \cap\left(\Omega \backslash \bar{C}_{\delta}\right)\right)}+\left\|\tilde{p}_{\delta}\right\|_{H_{\mu}^{1}\left(\tilde{\Omega}_{\Omega} \cap\left(\Omega \backslash \bar{C}_{\delta}\right)\right)} \\
& \leq c\left(\left\|\hat{v}_{\delta}\right\|_{H_{-\mu}^{2}\left(\mathbb{R}_{+}^{3} \backslash \bar{C}_{+\delta}\right)}+\left\|\hat{p}_{\delta}\right\|_{H_{-\mu}^{1}\left(\mathbb{R}_{+}^{3} \backslash \bar{C}_{+\delta}\right)}\right) \\
& \leq c\left(\left\|V_{\delta}\right\|_{H_{-\mu}^{2}\left(\mathbb{R}^{3} \backslash \bar{C}_{\delta}\right)}+\left\|P_{\delta}\right\|_{H_{-\mu}^{1}\left(\mathbb{R}^{3} \backslash \bar{C}_{\delta}\right)}\right) \\
& \leq c\left(\left\|H_{\delta}\right\|_{L_{2,-\mu}\left(\mathbb{R}^{3} \backslash \bar{C}_{\delta}\right)}+\left\|K_{\delta}\right\|_{H_{-\mu}^{1}\left(\mathbb{R}^{3} \backslash \bar{C}_{\delta}\right)}\right) \\
& \leq c\left(\left\|\hat{F}_{\delta}\right\|_{L_{2,-\mu}\left(\mathbb{R}_{+}^{3} \backslash \bar{C}_{+\delta}\right)}+\left\|\hat{G}_{\delta}\right\|_{H_{-\mu}^{1}\left(\mathbb{R}_{+}^{3} \backslash \bar{C}_{+\delta}\right)}\right) \\
& \leq c \frac{\delta_{0}}{\gamma}\left(\left\|p_{\delta}\right\|_{L_{2,-\mu-1}\left(\Omega \backslash \bar{C}_{\delta}\right)}+\left\|\nabla v_{\delta}\right\|_{L_{2,-\mu-1}\left(\Omega \backslash \bar{C}_{\delta}\right)}+\left\|v_{\delta}\right\|_{L_{2,-\mu-2}\left(\Omega \backslash \bar{C}_{\delta}\right)}\right) \\
& \quad+c\left(\left\|f_{\delta}\right\|_{L_{2,-\mu}\left(\Omega \backslash \bar{C}_{\delta}\right)}+\left\|g_{\delta}\right\|_{H_{-\mu}^{1}\left(\Omega \backslash \bar{C}_{\delta}\right)}\right) .
\end{aligned}
$$

This ends the proof.

Localization of problem (4.2) near a point lying at some distance from $L$. Let $x^{0} \in \Omega_{*}$ be such that $\operatorname{dist}\left(x^{0}, L\right)>R / 2$. We can assume that $x^{0} \in \tilde{\Omega}$, where $\tilde{\Omega} \subset \Omega_{*}$ is a cylinder such that $\tilde{\Omega} \cap \bar{C}_{\delta}=\emptyset$, and we choose a function $\zeta \in C_{0}^{\infty}(\tilde{\Omega})$ with the same properties as in the case of $x^{0} \in L \cap \Omega_{*}$.

Let $\tilde{v}_{\delta}=v_{\delta} \zeta, \tilde{p}_{\delta}=p_{\delta} \zeta$. Then $\left(\tilde{v}_{\delta}, \tilde{p}_{\delta}\right)$ satisfies problem $(4.10)$ in $\tilde{\Omega}=$ $\left(\Omega_{*} \backslash \bar{C}_{\delta}\right) \cap \tilde{\Omega}$. By extending by zero the data and $\tilde{v}_{\delta}, \tilde{p}_{\delta}$ to $\mathbb{R}^{3}$, the problem is reduced to a problem in $\mathbb{R}^{3}$.

LEMMA 4.4. Let the assumptions of Lemma 4.2 be satisfied. Then the solution $\left(\tilde{v}_{\delta}, \tilde{p}_{\delta}\right)$ of problem (4.10) in $\tilde{\Omega}$ satisfies the estimate

$$
\left\|\tilde{v}_{\delta}\right\|_{H_{-\mu}^{2}(\tilde{\Omega})}+\left\|\tilde{p}_{\delta}\right\|_{H_{-\mu}^{1}(\tilde{\Omega})} \leq c\left(\left\|f_{\delta}\right\|_{L_{2,-\mu}\left(\Omega \backslash \bar{C}_{\delta}\right)}+\left\|g_{\delta}\right\|_{H_{-\mu}^{1}\left(\Omega \backslash \bar{C}_{\delta}\right)}\right),
$$

where $c>0$ is independent of $\delta$.

Proof. Assume that $\operatorname{dist}(\tilde{\Omega}, L)>R / 4$. Then estimate (4.14) follows immediately as a consequence of the equivalence of $H_{-\mu}^{k}$ and $H^{k}(k=1,2)$ norms in this case and the regularity theory for the Stokes system in the usual Sobolev spaces (see [2, 6]) as well as Lemma 4.1.

The case of $x^{0} \in \partial \Omega_{*}, x^{0} \notin L$ can be treated as the previous case, i.e. straightening $\partial \Omega_{*}$ in a neighbourhood of $x^{0}$ we can reduce the problem to a 
problem in the halfspace $\mathbb{R}_{+}^{3}$. Then using the same argument as in the proof of Lemma 4.4 we can formulate the lemma below.

LEMMA 4.5. Let the assumptions of Lemma 4.3 hold. Then the solution $\left(\tilde{v}_{\delta}, \tilde{p}_{\delta}\right)$ of problem (4.11) in a domain $\tilde{\Omega} \cap \Omega_{*}$, where $\tilde{\Omega} \cap \partial \Omega_{*} \neq 0$, $\operatorname{dist}(\tilde{\Omega}, L)>R / 4$ and $\operatorname{diam} \tilde{\Omega}=\lambda(\lambda$ is sufficiently small) satisfies the estimate

$$
\left\|\tilde{v}_{\delta}\right\|_{H_{-\mu}^{1}(\tilde{\Omega} \cap \Omega)}+\left\|\tilde{p}_{\delta}\right\|_{H_{-\mu}^{1}(\tilde{\Omega} \cap \Omega)} \leq c\left(\left\|f_{\delta}\right\|_{L_{2,-\mu}\left(\Omega \backslash \bar{C}_{\delta}\right)}+\left\|g_{\delta}\right\|_{H_{-\mu}^{1}\left(\Omega \backslash \bar{C}_{\delta}\right)}\right),
$$

where $c>0$ is independent of $\delta$.

As a consequence of Lemmas 4.2-4.5 we get

Corollary 4.6. There is a solution $\left(v_{\delta}, p_{\delta}\right) \in H^{2}\left(\Omega \backslash \bar{C}_{\delta}\right) \times H^{1}\left(\Omega \backslash \bar{C}_{\delta}\right)$ satisfying inequality (4.9) with $c>0$ independent of $\delta$.

Proof. Let $\left\{\zeta_{i}\right\}$ be a partition of unity associated with a covering $\left\{\tilde{\Omega}_{i}\right\}$ of $\Omega_{*}$ by cylinders and assume that $\sup _{i} \operatorname{diam} \tilde{\Omega}_{i}=\lambda$, where $\lambda$ is sufficiently small.

Let $\left(\tilde{v}_{\delta}^{i}, \tilde{p}_{\delta}^{i}\right)=\left(v_{\delta} \zeta_{i}, p_{\delta} \zeta_{i}\right)$ (where $p_{\delta}$ satisfies $\left.(4.3)\right)$ be one of the four localized problems. Then $v_{\delta}=\sum_{i} \tilde{v}_{\delta}^{i}, p_{\delta}=\sum_{i} \tilde{p}_{\delta}$ is the solution of (4.2) satisfying the inequality

$$
\begin{aligned}
& \left\|v_{\delta}\right\|_{H_{-\mu}^{2}\left(\Omega \backslash \bar{C}_{\delta}\right)}+\left\|p_{\delta}\right\|_{H_{-\mu}^{1}\left(\Omega \backslash \bar{C}_{\delta}\right)} \\
& \leq c \frac{\delta_{0}}{\gamma}\left(\left\|p_{\delta}\right\|_{L_{2,-\mu-1}\left(\Omega \backslash \bar{C}_{\delta}\right)}+\left\|\nabla v_{\delta}\right\|_{L_{2,-\mu-1}\left(\Omega \backslash \bar{C}_{\delta}\right)}+\left\|v_{\delta}\right\|_{L_{2,-\mu-2}\left(\Omega \backslash \bar{C}_{\delta}\right)}\right) \\
& \quad+c\left(\left\|f_{\delta}\right\|_{L_{2,-\mu}\left(\Omega \backslash \bar{C}_{\delta}\right)}+\left\|g_{\delta}\right\|_{H_{-\mu}^{1}\left(\Omega \backslash \bar{C}_{\delta}\right)}\right) .
\end{aligned}
$$

Therefore, assuming that $\delta_{0}$ is sufficiently small we get inequality (4.9).

Now, we can finish the proof of the Main Theorem.

Proof of the Main Theorem. Let $\left(v_{\delta}, p_{\delta}\right)$ be a solution of (4.2) satisfying (4.9). In particular, $\left(v_{\delta}, p_{\delta}\right)$ is a weak solution satisfying the identities

$$
\int_{\Omega \backslash \bar{C}_{\delta}} \mathbb{D} v_{\delta}: \mathbb{D} \psi_{1} d x+\int_{\Omega \backslash \bar{C}_{\delta}} p_{\delta} \operatorname{div} \psi_{1} d x=\int_{\Omega \backslash \bar{C}_{\delta}} f_{\delta} \cdot \psi_{1} d x-\int_{\Omega \backslash \bar{C}_{\delta}} \nabla g_{\delta} \cdot \psi_{1} d x
$$

for every $\psi_{1} \in H^{1}\left(\Omega \backslash \bar{C}_{\delta}\right)$ with $\psi_{1}=0$ on $\left\{x \in \Omega:\left|x^{\prime}\right|=\delta\right\}$ and

$$
\int_{\Omega \backslash \bar{C}_{\delta}} \operatorname{div} v_{\delta} \psi_{2} d x=\int_{\Omega \backslash \bar{C}_{\delta}} g_{\delta} \psi_{2} d x
$$

for every $\psi_{2} \in H^{1}\left(\Omega \backslash \bar{C}_{\delta}\right)$ with $\psi_{2}=0$ on $\left\{x \in \Omega:\left|x^{\prime}\right|=\delta\right\}$. Extending the functions $v_{\delta}, p_{\delta}, f_{\delta}, g_{\delta}$ by zero to the functions $\bar{v}_{\delta} \in H_{-\mu-1}^{1}(\Omega)$ with $\bar{v}_{\delta, x_{i} x_{3}} \in L_{2,-\mu}(\Omega), i=1,2,3, \bar{p}_{\delta} \in L_{2,-\mu-1}(\Omega)$ with $\bar{p}_{\delta, x_{3}} \in L_{2,-\mu}(\Omega)$, $\bar{g}_{\delta} \in H_{-\mu}^{1}(\Omega)$, next extending $\psi_{1}, \psi_{2}$ by zero to $\bar{\psi}_{1}, \bar{\psi}_{2}$ and assuming that $\bar{\psi}_{1}=\phi_{1}\left|x^{\prime}\right|^{-2 \mu}, \bar{\psi}_{2}=\phi_{2}\left|x^{\prime}\right|^{-2 \mu}$ where $\phi_{1} \in H^{1}(\Omega), \phi_{1}=0$ on $\{x \in \Omega$ : 
$\left.\left|x^{\prime}\right|=\delta\right\}, \phi_{2} \in H^{1}(\Omega), \phi_{2}=0$ on $\left\{x \in \Omega:\left|x^{\prime}\right|=\delta\right\} \quad\left(\phi_{1}\right.$ and $\phi_{2}$ vanish in $C_{\delta}$ ) we find that the following identities hold:

$$
-2 \mu \int_{\Omega} \bar{p}_{\delta} \phi_{1} \cdot \nabla\left|x^{\prime}\right|\left|x^{\prime}\right|^{-2 \mu-1} d x=\int_{\Omega} \bar{f}_{\delta} \cdot \phi_{1}\left|x^{\prime}\right|^{-2 \mu} d x-\int_{\Omega} \nabla \bar{g}_{\delta} \cdot \phi_{1}\left|x^{\prime}\right|^{-2 \mu} d \mu
$$

for every function $\phi_{1} \in H^{1}(\Omega)$ vanishing in $C_{\delta}$ and

$$
\int_{\Omega} \operatorname{div} \bar{v}_{\delta} \phi_{2}\left|x^{\prime}\right|^{-2 \mu} d x=\int_{\Omega} \bar{g}_{\delta} \phi_{2}\left|x^{\prime}\right|^{-2 \mu} d x
$$

for every $\phi_{2} \in H^{1}(\Omega)$ vanishing in $C_{\delta}$.

Moreover, by estimate (4.9) the function $\left(\bar{v}_{\delta}, \bar{p}_{\delta}\right)$ satisfies

$$
\begin{array}{r}
\left\|\bar{v}_{\delta}\right\|_{H_{-\mu-1}^{1}(\Omega)}+\sum_{i=1}^{3}\left\|\bar{v}_{\delta, x_{i} x_{3}}\right\|_{L_{2,-\mu}(\Omega)}+\left\|\bar{p}_{\delta}\right\|_{L_{2,-\mu-1}(\Omega)} \\
+\left\|\bar{p}_{\delta, x_{3}}\right\|_{L_{2,-\mu}(\Omega)}+\left\|\bar{v}_{\delta}\right\|_{H_{-\mu}^{2}\left(\Omega_{R / 2}\right)}+\left\|\bar{p}_{\delta}\right\|_{H_{-\mu}^{1}\left(\Omega_{R / 2}\right)} \\
\quad \leq c\left(\left\|\bar{f}_{\delta}\right\|_{L_{2,-\mu}(\Omega)}+\left\|\bar{g}_{\delta}\right\|_{H_{-\mu}^{1}(\Omega)}\right)
\end{array}
$$

where $c>0$ does not depend on $\delta, \Omega_{R / 2}=\{x \in \Omega: \operatorname{dist}(x, L)>R / 2\}$.

Now, our aim is to show that $\left(\bar{v}_{\delta}, \bar{p}_{\delta}\right) \in H_{-\mu}^{2}(\Omega) \times H_{-\mu}^{1}(\Omega)$ and the norm of $\left(\bar{v}_{\delta}, \bar{p}_{\delta}\right)$ is bounded by the right-hand side of (4.17).

Let $x^{0} \in L \cap \Omega_{*}$ be an arbitrary point and consider the localized problem (4.10). Next, let $\left(\tilde{v}_{\delta}, \tilde{p}_{\delta}\right)$ be the solution of problem (4.12) and denote by $\left(\overline{\tilde{v}}_{\delta}, \overline{\tilde{p}}_{\delta}\right)$ the extension of $\left(\tilde{v}_{\delta}, \tilde{p}_{\delta}\right)$ by zero onto $\mathbb{R}^{3}$. Then $\overline{\tilde{v}}_{\delta}=\overline{v_{\delta} \zeta}=\bar{v}_{\delta} \zeta \equiv \tilde{\bar{v}}_{\delta}$, $\overline{\tilde{p}}_{\delta}=\overline{p_{\delta} \zeta}=\bar{p}_{\delta} \zeta=\tilde{\bar{p}}_{\delta}$ and by using the same argument as in the proof of Lemma 2.4 we conclude that $\left(\tilde{\bar{v}}_{\delta}, \tilde{\bar{p}}_{\delta}\right) \in L_{2}\left(\mathbb{R} ; H_{-\mu}^{2}\left(\mathbb{R}^{2}\right)\right) \cap L_{2}\left(\mathbb{R} ; H_{-\mu}^{1}\left(\mathbb{R}^{2}\right)\right)$ and $\left(\tilde{\bar{v}}_{\delta}, \tilde{\bar{p}}_{\delta}\right)$ satisfies the following two-dimensional system for a.e. $x_{3} \in \mathbb{R}$ :

$$
\begin{array}{ll}
-\Delta^{\prime} \tilde{\bar{v}}_{\delta}^{\prime}+\nabla^{\prime} \tilde{\bar{p}}_{\delta}=\overline{\tilde{f}}_{\delta}^{\prime}-\tilde{\bar{v}}_{\delta, x_{3} x_{3}}^{\prime}-2 \nabla \bar{v}_{\delta}^{\prime} \nabla \zeta & \\
-\bar{v}_{\delta}^{\prime} \Delta \zeta+\bar{p}_{\delta} \nabla^{\prime} \zeta \equiv \overline{\tilde{F}}_{\delta}^{\prime} & \text { in } \mathbb{R}^{2}, \\
\tilde{\bar{v}}_{\delta 1, x_{1}}+\tilde{\bar{v}}_{\delta 2, x_{2}}=-\tilde{\bar{v}}_{\delta 3, x_{3}}+\overline{\tilde{g}}_{\delta}+\bar{v}_{\delta} \cdot \nabla \zeta \equiv \overline{\tilde{G}}_{\delta} & \text { in } \mathbb{R}^{2}, \\
-\Delta^{\prime} \tilde{\bar{v}}_{\delta 3}=\overline{\tilde{f}}_{\delta 3}-\tilde{\bar{v}}_{\delta 3, x_{3} x_{3}}-\tilde{\bar{p}}_{\delta, x_{3}}-2 \nabla \bar{v}_{\delta 3} \nabla \zeta & \\
-\bar{v}_{\delta 3} \Delta \zeta+\bar{p}_{\delta} \zeta_{, x_{3}} \equiv \overline{\tilde{F}}_{\delta 3}, &
\end{array}
$$

where $\Delta^{\prime}=\partial_{x_{1}}^{2}+\partial_{x_{2}}^{2}, \nabla^{\prime}=\left(\partial_{x_{1}}, \partial_{x_{2}}\right), h^{\prime}=\left(h_{1}, h_{2}\right), h \in\left\{\tilde{\tilde{v}}_{\delta}, \bar{v}_{\delta}, \overline{\tilde{F}}_{\delta}\right\}$. 
Moreover, by Lemmas 2.1-2.2,

$$
\begin{aligned}
& \left\|\tilde{\bar{v}}_{\delta}^{\prime}\right\|_{L_{2}\left(\mathbb{R} ; H_{-\mu}^{2}\left(\mathbb{R}^{2}\right)\right)}+\left\|\tilde{\bar{p}}_{\delta}\right\|_{L_{2}\left(\mathbb{R} ; H_{-\mu}^{1}\left(\mathbb{R}^{2}\right)\right)} \\
\leq & c\left(\left\|\overline{\tilde{F}}_{\delta}^{\prime}\right\|_{L_{2,-\mu}\left(\mathbb{R}^{3}\right)}+\left\|\overline{\tilde{G}}_{\delta}\right\|_{H_{-\mu}^{1}\left(\mathbb{R}^{3}\right)}\right) \\
\leq & c\left(\left\|\bar{f}_{\delta}^{\prime}\right\|_{L_{2,-\mu}(\Omega)}+\left\|\tilde{\bar{v}}_{\delta, x_{3} x_{3}}^{\prime}\right\|_{L_{2,-\mu}(\Omega)}+\left\|\nabla \bar{v}_{\delta}\right\|_{L_{2,-\mu}(\Omega)}+\left\|\bar{v}_{\delta}\right\|_{L_{2,-\mu}(\Omega)}\right. \\
& \left.+\left\|\bar{p}_{\delta}\right\|_{L_{2,-\mu}(\Omega)}+\left\|\bar{g}_{\delta}\right\|_{H_{-\mu}^{1}(\Omega)}\right)
\end{aligned}
$$

and

$$
\begin{aligned}
& \left\|\tilde{\bar{v}}_{\delta 3}\right\|_{L_{2}\left(\mathbb{R} ; H_{-\mu}^{2}\left(\mathbb{R}^{2}\right)\right)} \leq c\left\|\overline{\tilde{F}}_{\delta 3}\right\|_{L_{2,-\mu}\left(\mathbb{R}^{3}\right)} \\
\leq & c\left(\left\|\bar{f}_{\delta 3}\right\|_{L_{2,-\mu}(\Omega)}+\left\|\tilde{\bar{v}}_{\delta 3, x_{3} x_{3}}\right\|_{L_{2,-\mu}(\Omega)}\right. \\
& \left.+\left\|\bar{p}_{\delta, x_{3}}\right\|_{L_{2,-\mu}(\Omega)}+\left\|\nabla \bar{v}_{\delta 3}\right\|_{L_{2,-\mu}(\Omega)}+\left\|\bar{v}_{\delta 3}\right\|_{L_{2,-\mu}(\Omega)}+\left\|\bar{p}_{\delta}\right\|_{L_{2,-\mu}(\Omega)}\right),
\end{aligned}
$$

where $c>0$ does not depend on $\delta$.

Using estimate (4.17) in (4.19)-(4.20) we get

$$
\left\|\tilde{\bar{v}}_{\delta}\right\|_{L_{2}\left(\mathbb{R} ; H_{-\mu}^{2}\left(\mathbb{R}^{2}\right)\right)}+\left\|\tilde{\bar{p}}_{\delta}\right\|_{L_{2}\left(\mathbb{R} ; H_{-\mu}^{1}\left(\mathbb{R}^{2}\right)\right)} \leq c\left(\left\|\bar{f}_{\delta}\right\|_{L_{2,-\mu}(\Omega)}+\left\|\bar{g}_{\delta}\right\|_{H_{-\mu}^{1}(\Omega)}\right) .
$$

In the case of $x^{0} \in L \cap \partial \Omega_{*}$ we also consider problem (4.18) but for almost all $x_{3}=y_{3} \in \mathbb{R}_{+}$. Then, by using the same argument as above we get

$$
\begin{aligned}
\left\|\tilde{\bar{v}}_{\delta}\right\|_{L_{2}\left(\mathbb{R}_{+} ; H_{-\mu}^{2}\left(\mathbb{R}^{2}\right)\right)}+\left\|\tilde{\bar{p}}_{\delta}\right\|_{L_{2}\left(\mathbb{R}_{+} ; H_{-\mu}^{1}\left(\mathbb{R}^{2}\right)\right)} & \leq c\left(\left\|\bar{f}_{\delta}\right\|_{L_{2,-\mu}(\Omega)}+\left\|\bar{g}_{\delta}\right\|_{H_{-\mu}^{1}(\Omega)}\right) .
\end{aligned}
$$

Now, estimates (4.17) and (4.21)-(4.22) yield the inequality

$$
\left\|\bar{v}_{\delta}\right\|_{H_{-\mu}^{2}(\Omega)}+\left\|\bar{p}_{\delta}\right\|_{H_{-\mu}^{1}(\Omega)} \leq c\left(\left\|\bar{f}_{\delta}\right\|_{L_{2,-\mu}(\Omega)}+\left\|\bar{g}_{\delta}\right\|_{H_{-\mu}^{1}(\Omega)}\right) .
$$

Hence, by the completeness of $H_{-\mu}^{1}(\Omega)$ and $L_{2,-\mu}(\Omega)$ there exists $(v, p) \in$ $H_{-\mu}^{2}(\Omega) \times H_{-\mu}^{1}(\Omega)$ such that

$$
\begin{array}{ll}
\bar{v}_{\delta} \rightarrow v & \text { in } H_{-\mu}^{2}(\Omega), \\
\bar{p}_{\delta} \rightarrow p & \text { in } H_{-\mu}^{1}(\Omega) .
\end{array}
$$

By (4.1) and the definition of $f_{\delta}$ the functions $v$ and $p$ satisfy identities (4.15)-(4.16) for every $\phi_{1} \in H_{-\mu}^{1}(\Omega)$ and $\phi_{2} \in H_{-\mu}^{1}(\Omega)$, respectively. Moreover, inequality (1.5) holds. This ends the proof.

Acknowledgments. This research was partially supported by MNiSW grant no. 1P03A 02130.

\section{References}

[1] R. R. Coifman and C. Fefferman, Weighted norm inequalities for maximal functions and singular integrals, Studia Math. 60 (1974), 241-250. 
[2] O. A. Ladyzhenskaya, The Mathematical Theory of Viscous Incompressible Flow, Nauka, Moscow, 1970 (in Russian).

[3] O. A. Ladyzhenskaya and V. A. Solonnikov, On some problems of vector analysis and generalized formulations of boundary problems for Navier-Stokes equations, Zap. Nauchn. Sem. LOMI 59 (1976), 81-116 (in Russian).

[4] V. A. Solonnikov, A priori estimates for some boundary problems, Dokl. Akad. Nauk SSSR 138 (1961), 781-784 (in Russian).

[5] - On differential properties of a solution of the first boundary problem for the nonstationary Navier-Stokes system, Trudy MIAN SSSR 73 (1964), 221-291 (in Russian).

[6] - On general boundary value problems for elliptic system of the Douglis-Nirenberg type, I, Izv. Akad. Nauk SSSR Ser. Mat. 28 (1964), 665-706; II, Trudy MIAN SSSR 92 (1966), 233-297 (in Russian).

[7] - On the solvability of the second initial-boundary value problem for the linear nonstationary Navier-Stokes equations, Zap. Nauchn. Sem. LOMI 69 (1977), 200218 (in Russian).

[8] -, Solvability of a three-dimensional problem with free boundary for the stationary Navier-Stokes equations, Zap. Nauchn. Sem. LOMI 84 (1979), 252-285 (in Russian).

[9] V. A. Solonnikov and V. E. Shchadilov, On a boundary-value problem for the stationary Navier-Stokes system, Trudy MIAN SSSR 125 (1973), 196-210 (in Russian).

[10] I. I. Vorovich and V. I. Yudovich, Stationary motion of a viscous incompressible fluid, Mat. Sb. 53 (1961), 393-428 (in Russian).

[11] W. M. Zajączkowski, Existence of solutions to the nonstationary Stokes system in $H_{-\mu}^{2,1}, \mu \in(0,1)$, in a domain with a distinguished axis. Part 1. Existence near the axis in 2d, Appl. Math. (Warsaw) 34 (2007), 121-141.

[12] - Estimates for solutions to the nonstationary Stokes system in $H_{-\mu}^{2,1}, \mu \in(0,1)$, in a domain with a distinguished axis. Part 2. Estimate in the 3d case, ibid. 34 (2007), 143-167.

[13] —, Nonstationary Stokes system in weighted Sobolev spaces, to appear.

Ewa Zadrzyńska

Faculty of Mathematics and Information Sciences

Warsaw University of Technology

Pl. Politechniki 1

00-661 Warszawa, Poland
Wojciech M. Zajączkowski

Institute of Mathematics

Polish Academy of Sciences

Sniadeckich 8

00-956 Warszawa, Poland

E-mail: wz@impan.pl

Institute of Mathematics and Cryptology

Cybernetics Faculty

Military University of Technology

Kaliskiego 2

00-908 Warszawa, Poland

Received on 10.10.200\%;

revised version on 7.10 .2009 